\title{
On the cell-dependent vibrations and wave propagation in uniperiodic cylindrical shells
}

Received: 13 August 2019 / Accepted: 27 September 2019 / Published online: 11 October 2019

(C) The Author(s) 2019

\begin{abstract}
The objects of consideration are thin linearly elastic Kirchhoff-Love-type circular cylindrical shells having a periodically micro-heterogeneous structure in circumferential direction (uniperiodic shells). The aim of this contribution is to study certain problems of micro-vibrations and of wave propagation related to microfluctuations of displacement field caused by a periodic structure of the shells. These micro-dynamic problems will be analysed in the framework of a certain mathematical averaged model derived by means of the combined modelling procedure. The combined modelling includes both the asymptotic and the tolerance non-asymptotic modelling techniques, which are conjugated with themselves under special conditions. Contrary to the starting exact shell equations with highly oscillating, non-continuous and periodic coefficients, governing equations of the combined model have constant coefficients depending also on a cell size. Hence, this model takes into account the effect of a microstructure size on the dynamic behaviour of the shells (the length-scale effect). It will be shown that the micro-periodic heterogeneity of the shells leads to cell-depending micro-vibrations and to exponential waves as well as to dispersion effects, which cannot be analysed in the framework of the asymptotic models commonly used for investigations of vibrations and wave propagation in the periodic structures.
\end{abstract}

Keywords Uniperiodic shells · Asymptotic and tolerance modelling · Micro-dynamics

Communicated by Andreas Öchsner.

B. Tomczyk

Department of Civil Engineering, Warsaw University of Life Sciences, Nowoursynowska Str. 166, 02-787 Warsaw, Poland E-mail: barbara_tomczyk@sggw.pl

M. Gołąbczak (凶)

Institute of Machine Tools and Production Engineering, Lodz University of Technology, Stefanowskiego Str. 1/15, 90-924 Lodz, Poland

E-mail: marcin.golabczak@p.lodz.pl

A. Litawska

Department of Structural Mechanics, Lodz University of Technology, Politechniki 6, 90-924 Lodz, Poland

E-mail: a.litawska@gmail.com

A. Gołąbczak

State Higher Vocational School in Włocławek, 3 Maja Str. 17, 87-800 Włocławek, Poland

E-mail: andrzej.golabczak@p.lodz.pl 


\section{Introduction}

Thin linearly elastic Kirchhoff-Love-type circular cylindrical shells with a periodically micro-inhomogeneous structure (a periodically varying thickness and/or periodically varying elastic and inertial properties) in circumferential direction are analysed. Shells of this kind are termed uniperiodic. At the same time, the shells have constant structure in axial direction. The shells under consideration are composed of a large number of identical elements, and every such element, called a periodicity cell, can be treated as a thin shell. It means that the period of inhomogeneity is very large compared with the maximum shell thickness and very small as compared to the midsurface curvature radius as well as the length dimension of the shell midsurface in the periodicity direction. As examples, we can mention cylindrical shells with periodically spaced stiffeners as shown in Fig. 1 or made of two kinds of periodically distributed materials as shown in Fig. 2.

It should be noted that in the general case, on the shell midsurface we deal with not periodic but locally periodic structure. By a locally periodic shell we mean a shell which, in small subregions of the shell midsurface, can be approximately regarded as periodic. Hence, a locally periodic shell is made of a large number of not identical, but similar elements. However, for cylindrical shells the Gaussian curvature is equal to zero, and hence, on the developable cylindrical surface we can separate a cell which can be referred to as the representative cell for the whole shell midsurface. It means that on cylindrical surface, we deal not with locally periodic but with a periodic structure.

Dynamic problems of periodic shells are described by partial differential equations with highly oscillating, periodic and non-continuous coefficients. Thus, these equations are too complicated to be applied to the investigations of engineering problems. To obtain averaged equations with constant coefficients, various approximate modelling procedures for shells of this kind have been proposed. Periodic cylindrical shells (plates) are usually described using homogenized models derived by means of asymptotic methods. These models from a formal

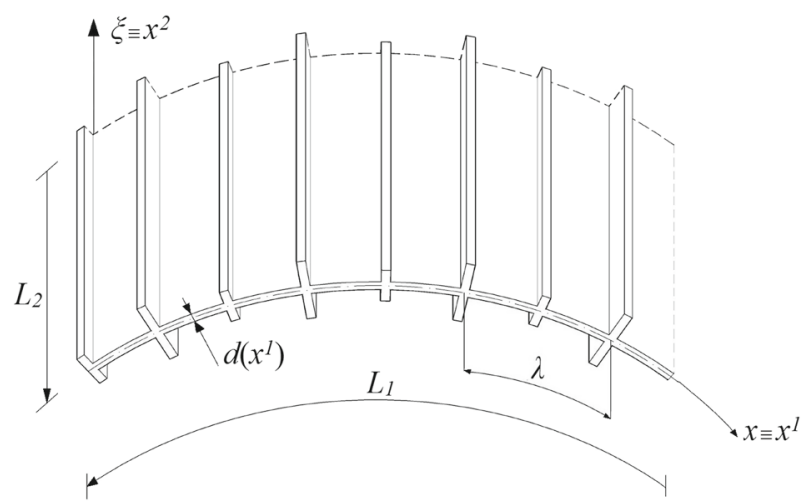

Fig. 1 Fragment of the shell with two families of uniperiodically spaced stiffeners

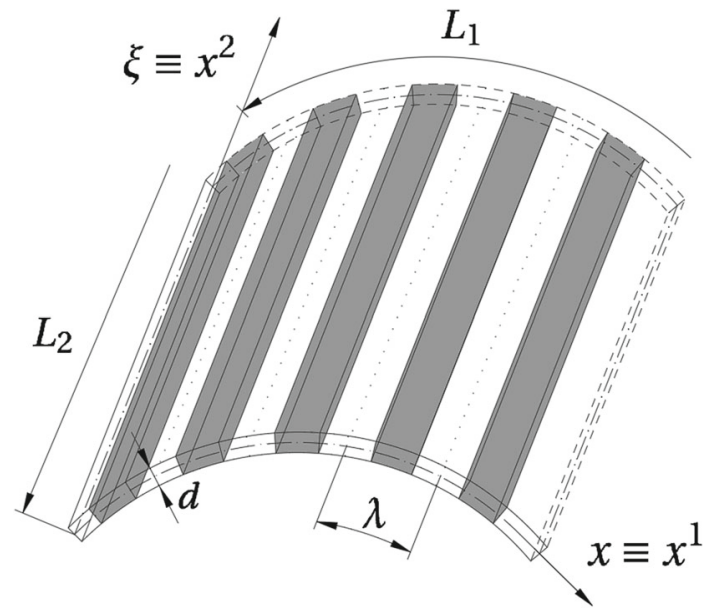

Fig. 2 Fragment of the shell made of uniperiodically distributed two component materials 
point of view represent certain equivalent structures with constant or slowly varying stiffnesses and averaged mass densities. From the extensive list on this subject, we can mention monograph by Lewiński and Telega [1], where asymptotic modelling of plates, laminates and shells is discussed. Unfortunately, in the models of this kind the effect of a periodicity cell length dimensions (called the length-scale effect) on the overall shell behaviour is neglected.

This effect can be taken into account using the modified couple stress-based theories of continuous media. We mention here paper by Awrejcewicz et al. [2], where mathematical model for the analysis of static and dynamic problems of micro/nano-beams is derived and discussed; the size-dependent model equations are formulated on the basis of the Grigolyuk-Chulkov hypotheses and the modified couple stress theory.

The length-scale effect can be bearing in mind applying the multiscale-multifield models derived from a non-classical (generalized) continuum formulation. These models take into account the microstructure size by means of microdisplacement variables added to the standard macrodisplacements and of material internal length parameters, cf., e.g. Settimi et al. [3], where the length-scale effect on the dynamic properties of a composite microcracked elastic bar is studied; the internal parameters represent here density and length of microcracks.

Some numerical approaches are also proposed to study the size effects in mechanical problems for microheterogeneous structures. As example, we can mention here the paper by Hassani et al. [4], where the sizedependent variational differential quadrature procedure is combined with the finite element method into a new technique. In this paper, by considering several numerical examples, it has been shown that the proposed size-dependent formulation and numerical solution approach have a good performance to study the large deformations of hyperelastic microstructured bodies.

The length-scale effect can be also taken into account using the non-asymptotic-tolerance averaging technique, cf. Woźniak and Wierzbicki [5], Woźniak et al. [6,7]. Some applications of this method to the modelling of mechanical and thermomechanical problems for various periodic structures are shown in many works. The extended list of publications on this topic can be found in [5-7]. We mention here monograph by Tomczyk [8], where the length-scale effect in dynamics and stability of periodic cylindrical shells is investigated, paper by Marczak and Jędrysiak [9], where vibrations of periodic three-layered plates with inert core are studied and papers by Tomczyk and Litawska [10,11], where certain extended co-called general tolerance and general asymptotic-tolerance models for the analysis of dynamic problems for periodic cylindrical shells are proposed and discussed. These general models are derived by means of a certain extended version of the tolerance modelling technique presented by Tomczyk and Woźniak [12].

In the last years, the tolerance modelling was adopted for mechanical and thermomechanical problems of functionally graded structures, e.g. for heat conduction in longitudinally graded structures by Ostrowski and Michalak [13], for thermoelasticity of transversally graded laminates by Pazera and Jędrysiak [14], for vibrations of functionally graded thin plates by Wirowski [15], for dynamics of transversally and longitudinally graded thin cylindrical shells by Tomczyk and Szczerba [16-18].

The aim of this note is to study certain problems of cell-depending vibrations and of long wave propagation related to micro-fluctuations of displacement field caused by a periodic structure of the shells. Note that we deal with long waves if condition $\lambda / L<<1$ holds, where $\lambda$ is the characteristic length dimension of the cell and $L$ is the wavelength. These micro-dynamic problems will be analysed in the framework of the combined asymptotic-tolerance model proposed in [8]. Governing equations of this averaged model have constant coefficients depending also on a microstructure size. An important advantage of this model is that it makes it possible to separate the macroscopic description of the modelling problem from its microscopic description. It will be shown that the periodic micro-heterogeneity of the shells leads to vibrations depending on a cell size and to exponential waves as well as to dispersion effects, which cannot be analysed in the framework of the asymptotic models commonly used for investigations of vibrations and wave propagation in the periodic shells under consideration. The new wave propagation speed depending on a cell size will be obtained and analysed.

It should be noted that this article is a certain continuation of papers by Tomczyk and Szczerba [16-18] and by Tomczyk and Litawska [10,11], in which some special dynamic problems for thin functionally graded cylindrical shells [16-18] and for shells with two-directional periodic structure in directions tangent to the shell midsurface (biperiodic shells) $[10,11]$ are analysed by applying the tolerance modelling technique. Note that in the non-asymptotic-tolerance approach, shells with one-directional periodic structure (uniperiodic shells) being objects of consideration in this work are not special cases of biperiodic shells. Model equations for uniperiodic shells are more complicated than those for biperiodic shells and contain a lot of length-scale terms which do not have counterparts in the equations for biperiodic shells. The occurrence of these terms is strictly 
related to the fact that the modelling physical reliability conditions for uniperiodic shells are less restrictive than those for biperiodic shells.

The periodic shells being objects of considerations in this paper are widely applied in civil engineering, most often as roof girders and bridge girders. They are also widely used as housings of reactors and tanks. Periodic shells having small length dimensions are elements of air-planes, ships and machines.

\section{Formulation of the problem: starting equations}

We assume that $x^{1}$ and $x^{2}$ are coordinates parametrizing the shell midsurface $M$ in circumferential and axial directions, respectively. We denote $x \equiv x^{1} \in \Omega \equiv\left(0, L_{1}\right)$ and $\xi \equiv x^{2} \in \Xi \equiv\left(0, L_{2}\right)$, where $L_{1}, L_{2}$ are length dimensions of $M$,cf. Figs. 1 and 2. Let $O \bar{x}^{1} \bar{x}^{2} \bar{x}^{3}$ stand for a Cartesian orthogonal coordinate system in the physical space $R^{3}$ and denote $\overline{\mathbf{x}} \equiv\left(\bar{x}^{1}, \bar{x}^{2}, \bar{x}^{3}\right)$. A cylindrical shell midsurface $M$ is given by $M \equiv\left\{\overline{\mathbf{x}} \in R^{3}: \overline{\mathbf{x}}=\overline{\mathbf{r}}\left(x^{1}, x^{2}\right),\left(x^{1}, x^{2}\right) \in \Omega \times \Xi\right\}$, where $\overline{\mathbf{r}}(\cdot)$ is the smooth invertible function such that $\partial \overline{\mathbf{r}} / \partial x^{1} \cdot \partial \overline{\mathbf{r}} / \partial x^{2}=0, \partial \overline{\mathbf{r}} / \partial x^{1} \cdot \partial \overline{\mathbf{r}} / \partial x^{1}=1, \partial \overline{\mathbf{r}} / \partial x^{2} \cdot \partial \overline{\mathbf{r}} / \partial x^{2}=1$. It means that on $M$, the orthonormal parametrization is introduced. Note that derivative $\partial \overline{\mathbf{r}} / \partial x^{\alpha}, \alpha=1,2$, should be understood as differentiation of each component of $\overline{\mathbf{r}}$, i.e. $\partial \overline{\mathbf{r}} / \partial x^{\alpha}=\left[\partial \bar{r}^{1} / \partial x^{\alpha}, \partial \bar{r}^{2} / \partial x^{\alpha}, \partial \bar{r}^{3} / \partial x^{\alpha}\right]$ for $\overline{\mathbf{r}}=\left[\bar{r}^{1}, \bar{r}^{2}, \bar{r}^{3}\right]$.

Sub- and superscripts $\alpha, \beta, \ldots$ run over 1,2 and are related to $x^{1}, x^{2}$, summation convention holds. Partial differentiation related to $x^{\alpha}$ is represented by $\partial_{\alpha}, \partial_{\alpha}=\partial / \partial x_{\alpha}$. Moreover, it is denoted $\partial_{\alpha \ldots \delta} \equiv \partial_{\alpha} \ldots \partial_{\delta}$. Let $a^{\alpha \beta}$ and $b_{\alpha \beta}$ stand for the midsurface first and second metric tensors, respectively. Under orthonormal parametrization introduced on $M, a^{\alpha \beta}$ is a unit tensor and components of tensor $b_{\alpha \beta}$ are: $b_{22}=b_{12}=b_{21}=0$, $b_{11}=-r^{-1}$. The time coordinate is denoted by $t \in \mathrm{I}=\left[t_{0}, t_{1}\right]$. Differentiation with respect to time is represented by the overdot. Let $d(x), r$ stand for the shell thickness and the midsurface curvature radius, respectively.

The basic cell $\Delta$ and an arbitrary cell $\Delta(x)$ with the centre at point $x \in \Omega_{\Delta}$ are defined by means of: $\Delta \equiv[-\lambda / 2, \lambda / 2], \Delta(x) \equiv x+\Delta, \quad x \in \Omega_{\Delta}, \Omega_{\Delta} \equiv\{x \in \Omega: \Delta(x) \subset \Omega\}$, where $\lambda$ is a cell length dimension in $x \equiv x^{1}$-direction. The microstructure length parameter $\lambda$ satisfies conditions: $\lambda / d_{\max }>>1, \quad \lambda / r<<1$ and $\lambda / L_{1}<<1$.

Setting $z \equiv z^{1} \in[-\lambda / 2, \lambda / 2]$, we assume that the cell $\Delta$ has a symmetry axis for $z=0$. It is also assumed that inside the cell the geometrical, elastic and inertial properties of the shell are described by even functions of argument $z$.

Denote by $u_{\alpha}=u_{\alpha}(x, \xi, t), w=w(x, \xi, t),(x, \xi, t) \in \Omega \times \Xi \times \mathrm{I}$, the shell displacements in directions tangent and normal to $M$, respectively. Elastic properties of the shells are described by shell stiffness tensors $D^{\alpha \beta \gamma \delta}(x), B^{\alpha \beta \gamma \delta}(x)$. Let $\mu(x)$ stand for a shell mass density per midsurface unit area. The external forces will be neglected.

The considerations are based on the well-known Kirchhoff-Love theory of thin elastic shells, cf. Kaliski [19].

In the framework of the shell theory under consideration, strain energy function $E(x, \xi, t),(x, \xi, t) \in$ $\Omega \times \Xi \times I$, related to midsurface $M$ has the form

$$
E=\frac{1}{2}\left(D^{\alpha \beta \gamma \delta} \varepsilon_{\alpha \beta} \varepsilon_{\gamma \delta}+B^{\alpha \beta \gamma \delta} \kappa_{\alpha \beta} \kappa_{\gamma \delta \delta}\right),
$$

where the membrane $\varepsilon_{\alpha \beta}(x, \xi, t)$ and curvature $\mathrm{K}_{\alpha \beta}(x, \xi, t)$ strain tensors are

$$
\varepsilon_{\alpha \beta}=\frac{1}{2}\left(\partial_{\beta} u_{\alpha}+\partial_{\alpha} u_{\beta}\right)-b_{\alpha \beta} w, \quad \kappa_{\alpha \beta}=-\partial_{\alpha \beta} w .
$$

The kinetic energy function $K(x, \xi, t),(x, \xi, t) \in \Omega \times \Xi \times \mathrm{I}$, related to midsurface $M$ is given by

$$
K=\frac{1}{2} \mu\left(\dot{u}_{\alpha} \dot{u}_{\beta} a^{\alpha \beta}+\dot{w} \dot{w}\right) .
$$

Let us introduce the action functional

$$
A\left(u_{\alpha}, w\right)=\int_{0}^{L_{1}} \int_{0}^{L_{2}} \int_{t_{0}}^{t_{1}} L\left(x, \xi, t, \partial_{\beta} u_{\alpha}, \dot{u}_{\alpha}, \partial_{\alpha \beta} w, w, \dot{w}\right) \mathrm{d} t \mathrm{~d} \xi \mathrm{d} x,
$$


with lagrangian $L$ being a highly oscillating function with respect to $x$. Here, under assumption that the external forces are neglected, lagrangian $L$ have the form

$$
L=K-E,
$$

where kinetic energy $K$ and strain energy $E$ are given above.

Substituting (1)-(3) into (5) and taking into account that $b_{22}=b_{12}=b_{21}=0$ and $b_{11}=-r^{-1}$, we arrive at Lagrange function (5) in the form

$$
\begin{aligned}
L= & -\frac{1}{2}\left(D^{\alpha \beta \gamma \delta} \partial_{\beta} u_{\alpha} \partial_{\delta} u_{\gamma}+2 r^{-1} D^{\alpha \beta 11} w \partial_{\beta} u_{\alpha}+r^{-2} D^{1111} w w\right. \\
& \left.+B^{\alpha \beta \gamma \delta} \partial_{\alpha \beta} w \partial_{\gamma \delta} w-\mu a^{\alpha \beta} \dot{u}_{\alpha} \dot{u}_{\beta}-\mu \dot{w}^{2}\right) .
\end{aligned}
$$

Under assumption that $\partial L / \partial\left(\partial_{\beta} u_{\alpha}\right)$ and $\partial L / \partial\left(\partial_{\alpha \beta} w\right)$ are continuous, from the principle of stationary action applied to $A\left(u_{\alpha}, w\right)$, we obtain the system of Euler-Lagrange equations, which can be written in an explicit form as

$$
\begin{aligned}
& \partial_{\beta}\left(D^{\alpha \beta \gamma \delta} \partial_{\delta} u_{\gamma}\right)+r^{-1} \partial_{\beta}\left(D^{\alpha \beta 11} w\right)-\mu a^{\alpha \beta} \ddot{u}_{\beta}=0, \\
& r^{-1} D^{\alpha \beta 11} \partial_{\beta} u_{\alpha}+\partial_{\alpha \beta}\left(B^{\alpha \beta \gamma \delta} \partial_{\gamma \delta} w\right)+r^{-2} D^{1111} w+\mu \ddot{w}=0 .
\end{aligned}
$$

Equations (7) coincide with the well-known governing equations of Kirchhoff-Love theory of thin elastic shells, cf. Kaliski [19]. For periodic shells, coefficients $D^{\alpha \beta \gamma \delta}(x), B^{\alpha \beta \gamma \delta}(x), \mu(x)$ of (6) and (7) are highly oscillating, non-continuous and periodic functions in $x$. Applying the combined asymptotic-tolerance modelling technique, the averaged model equations with constant coefficients depending also on a cell size were derived in [8]. The combined modelling given in the general form in Woźniak et al. [7] includes both the asymptotic and the tolerance non-asymptotic modelling techniques, which are conjugated with themselves under special conditions. Contrary to the starting exact shell equations with highly oscillating, non-continuous and periodic coefficients, governing equations of the combined model have constant coefficients depending also on a cell size. Hence, this model takes into account the effect of a microstructure size on the dynamic behaviour of the shells (the length-scale effect). Here, the combined model equations formulated in [8] will be used for investigations of two special micro-dynamic problems for cylindrical shell having constant thickness and made of two component linearly elastic isotropic materials periodically distributed in circumferential direction as shown in Fig. 2. The first of these problems deals with cell-depending micro-vibrations caused by a periodic structure of the shell. The second one deals with propagation of the long waves related to micro-fluctuations of axial displacements.

It has to be emphasized that these aforementioned special micro-dynamic problems can be studied in the framework of neither the asymptotic models nor the known commercial numerical models based on the finite element method.

To make the analysis more clear, in the next section the asymptotic-tolerance model for the shells under consideration will be reminded, following [8]. Moreover, the basic concepts and assumptions of the tolerance modelling technique and of the consistent asymptotic approach will be outlined, following $[7,8]$.

\section{Modelling procedure: asymptotic-tolerance model}

The combined modelling technique under consideration is realized in two steps. The first step is based on the consistent asymptotic modelling procedure $[7,8]$. The second one is realized by means of the tolerance non-asymptotic modelling technique $[7,8]$.

\subsection{Step 1. Consistent asymptotic modelling}

The fundamental concepts of the consistent asymptotic procedure are those of an averaging operation and fluctuation shape functions. In what follows, the above-mentioned concepts will be specified with respect to one-dimensional region $\Omega \equiv\left(0, L_{1}\right)$ defined in this contribution.

Let $f(x)$ be a function defined in $\bar{\Omega} \equiv\left[0, L_{1}\right]$, which is integrable and bounded in every cell $\Delta(x), x \in \Omega_{\Delta}$. The averaging operation of $f(\cdot)$ is defined by

$$
<f>(x) \equiv \frac{1}{|\Delta|} \int_{\Delta(x)} f(z) \mathrm{d} z, \quad z \in \Delta(x), \quad x \in \Omega_{\Delta} .
$$


It can be seen that if $f(\cdot)$ is $\Delta$-periodic, then $<f>$ is constant.

Denote by $\partial_{1}^{k}$ the $k$ th derivative of function defined in $\Omega$. Let $h(x)$ be a $\lambda$-periodic, highly oscillating function defined in $\bar{\Omega}=\left[0, L_{1}\right]$, which is continuous together with derivatives $\partial_{1}^{k} h, k=1, \ldots, R-1$, and has a continuous or piecewise continuous bounded derivative $\partial_{1}^{R} h$. Function $h(\cdot)$ will be called the fluctuation shape function of the Rth kind, $h(\cdot) \in F S^{R}(\Omega, \Delta)$, if it satisfies conditions: $h \in O\left(\lambda^{R}\right), \partial_{1}^{k} h \in O\left(\lambda^{R-k}\right), k=$ $1,2, \ldots, R, \quad\langle\mu h>=0$, where $\mu(x)$ is a shell mass density. Nonnegative integer $R$ is assumed to be specified in every problem under consideration.

The first step of the combined modelling is based on the consistent asymptotic averaging of lagrangian (6). To this end, we shall restrict considerations to displacement fields $u_{\alpha}=u_{\alpha}(z, \xi, t), w=w(z, \xi, t)$ defined in $\Delta(x) \times \Xi \times I, z \equiv z^{1} \in \Delta(x), x \in \Omega_{\Delta},(\xi, t) \in \Xi \times I$. Then, we replace $u_{\alpha}(z, \xi, t), w(z, \xi, t)$ by families of displacements $u_{\varepsilon \alpha}(z, \xi, t) \equiv u_{\alpha}(z / \varepsilon, \xi, t), w_{\varepsilon}(z, \xi, t) \equiv w(z / \varepsilon, \xi, t)$, where $\varepsilon=1 / m, m=1,2, \ldots,(\varepsilon$ is a small parameter), $z \in \Delta_{\varepsilon}(x), \quad \Delta_{\varepsilon} \equiv(-\varepsilon \lambda / 2, \varepsilon \lambda / 2)$ (scaled cell), $\Delta_{\varepsilon}(x) \equiv x+\Delta_{\varepsilon}, x \in \Omega_{\Delta_{\varepsilon}}$ (scaled cell with a centre at $x \in \Omega_{\Delta_{\varepsilon}}$. .

We introduce the consistent asymptotic decomposition of families of displacements $u_{\varepsilon \alpha}(z, \xi, t)$, $w_{\varepsilon}(z, \xi, t),(z, \xi, t) \in \Delta_{\varepsilon} \times \Xi \times I$

$$
\begin{aligned}
& u_{\varepsilon \alpha}(z, \xi, t) \equiv u_{\alpha}(z / \varepsilon, \xi, t)=u_{\alpha}^{0}(z, \xi, t)+\varepsilon h_{\varepsilon}(z) U_{\alpha}(z, \xi, t), \\
& w_{\varepsilon}(z, \xi, t) \equiv w(z / \varepsilon, \xi, t)=w^{0}(z, \xi, t)+\varepsilon^{2} g_{\varepsilon}(z) W(z, \xi, t) .
\end{aligned}
$$

Unknown functions $u_{\alpha}^{0}, U_{\alpha}$ in (9) are assumed to be continuous and bounded in $\Omega$ together with their first derivatives. Unknown functions $w^{0}, W$ in (9) are assumed to be continuous and bounded in $\Omega$ together with their derivatives up to the second order. Unknowns $u_{\alpha}^{0}, w^{0}$ and $U_{\alpha}^{\prime} W$ are called macrodisplacements and fluctuation amplitudes, respectively. They are independent of $\varepsilon$. This is the main difference between the asymptotic approach under consideration and approach which is used in the known homogenization theory, cf. Bensoussan et al. [20], Jikov et al. [21].

By $h_{\varepsilon}(z) \equiv h(z / \varepsilon) \in F S^{1}(\Omega, \Delta)$ and $g_{\varepsilon}(z) \equiv g(z / \varepsilon) \in F S^{2}(\Omega, \Delta)$ in (9) are denoted $\lambda$-periodic highly oscillating fluctuation shape functions depending on $\varepsilon$. The fluctuation shape functions are assumed to be known in every problem under consideration. They have to satisfy conditions: $h_{\varepsilon} \in O(\varepsilon \lambda), \lambda \partial_{1} h_{\varepsilon} \in O(\varepsilon \lambda)$, $g_{\varepsilon} \in O\left((\varepsilon \lambda)^{2}\right), \lambda \partial_{1} g_{\varepsilon} \in O\left((\varepsilon \lambda)^{2}\right), \quad \lambda^{2} \partial_{11} g_{\varepsilon} \in O\left((\varepsilon \lambda)^{2}\right),<\mu h_{\varepsilon}>=<\mu g_{\varepsilon}>0$. It has to be emphasized that $\partial_{1} h_{\varepsilon}(z) \equiv \frac{1}{\varepsilon} \partial_{1} h(z / \varepsilon), \quad \partial_{1} g_{\mathcal{\varepsilon}}(z) \equiv \frac{1}{\varepsilon} \partial_{1} g(z / \varepsilon), \quad \partial_{11} g_{\mathcal{\varepsilon}}(z) \equiv \frac{1}{\varepsilon^{2}} \partial_{11} g(z / \varepsilon)$.

We substitute the right-hand sides of (9) into (6) and take into account that under limit passage $\varepsilon \rightarrow 0$, terms depending on $\varepsilon$ can be neglected and every continuous and bounded function of argument $z \in \Delta_{\varepsilon}(x)$ tends to function of argument $x \in \bar{\Omega}$. Moreover, if $\varepsilon \rightarrow 0$ then by means of a property of the mean value, cf. [21], the obtained result tends weakly to the function being the averaged form of starting lagrangian (6) under consistent asymptotic decomposition (9). Then, applying the principle of stationary action we obtaingoverning equations of the consistent asymptotic model for the unperiodic shells under consideration. These equations consist of partial differential equations for macrodisplacements $u_{\alpha}^{0}, w^{0}$ coupled with linear algebraic equations for fluctuation amplitudes $U_{\alpha}, W$. After eliminating fluctuation amplitudes from the governing equations by means of

$$
\begin{aligned}
& U_{\gamma}=-\left(G^{-1}\right)_{\gamma \eta}\left[<\partial_{1} h D^{1 \eta \mu \vartheta}>\partial_{\vartheta} u_{\mu}^{0}+r^{-1}<\partial_{1} h D^{1 \eta 11}>w^{0}\right], \\
& W=-E^{-1}<\partial_{11} g B^{11 \gamma \delta}>\partial_{\gamma \delta} w^{0},
\end{aligned}
$$

where $G_{\alpha \gamma}=<D^{\alpha 1 \gamma 1}\left(\partial_{1} h\right)^{2}>, E=<B^{1111}\left(\partial_{11} g\right)^{2}>$, we arrive finally at the asymptotic model equations expressed only in macrodisplacements $u_{\alpha}^{0}, w^{0}$

$$
\begin{aligned}
& D_{h}^{\alpha \beta \gamma \delta} \partial_{\beta \delta} u_{\gamma}^{0}+r^{-1} D_{h}^{\alpha \beta 11} \partial_{\beta} w^{0}-<\mu>a^{\alpha \beta} \ddot{u}_{\beta}^{0}=0, \\
& B_{g}^{\alpha \beta \gamma \delta} \partial_{\alpha \beta \gamma \delta} w^{0}+r^{-1} D_{h}^{11 \gamma \delta} \partial_{\delta} u_{\gamma}^{0}+r^{-2} D_{h}^{1111} w^{0}+<\mu>\ddot{w}^{0}=0,
\end{aligned}
$$

where

$$
\begin{aligned}
& D_{h}^{\alpha \beta \gamma \delta} \equiv<D^{\alpha \beta \gamma \delta}>-<D^{\alpha \beta \eta 1} \partial_{1} h>\left(G^{-1}\right)_{\eta \zeta}<\partial_{1} h D^{1 \zeta \gamma \delta}> \\
& B_{g}^{\alpha \beta \gamma \delta} \equiv<B^{\alpha \beta \gamma \delta}>-<B^{\alpha \beta 11} \partial_{11} g>E^{-1}<\partial_{11} g B^{11 \gamma \delta}>.
\end{aligned}
$$


Since displacement fields $u_{\alpha}(x, \xi, t), w(x, \xi, t)$ have to be uniquely defined in $\Omega \times \Xi \times \mathrm{I}$, we conclude that $u_{\alpha}(x, \xi, t), w(x, \xi, t)$ have to take the form

$$
\begin{aligned}
& u_{\alpha}(x, \xi, t)=u_{\alpha}^{0}(x, \xi, t)+h(x) U_{\alpha}(x, \xi, t), \\
& w(x, \xi, t)=w^{0}(x, \xi, t)+g(x) W(x, \xi, t), \quad(x, \xi, t) \in \Omega \times \Xi \times I,
\end{aligned}
$$

with $U_{\alpha}, W$ given by (10). Tensors $D_{h}^{\alpha \beta \gamma \delta}, B_{g}^{\alpha \beta \gamma \delta}$ given by (12) are tensors of effective elastic moduli for the considered composite uniperiodic shells.

In contrast to starting equations (7) with discontinuous, highly oscillating and periodic coefficients, the asymptotic model equations (11) have coefficients constant but independent of the microstructure size $\lambda$. Hence, the above model is not able to describe the length-scale effect on the overall shell dynamics. That is why, the model derived in the first step of combined modelling is referred to as the macroscopic model for the problem under consideration.

Unknown macrodisplacements $u_{\alpha}^{0}, w^{0}$ and fluctuation amplitudes $U_{\alpha}, W$ must be continuously bounded in $\Omega$.

The resulting equations (11) are uniquely determined by the postulated a priori periodic fluctuations shape functions, $h(x) \in F S^{1}(\Omega, \Delta), h \in O(\lambda)$, and $g(x) \in F S^{2}(\Omega, \Delta), g \in O\left(\lambda^{2}\right)$, representing oscillations inside a cell. These functions can be derived from the periodic discretization of the cell using, for example, the finite element method or obtained as exact or approximate solutions to certain periodic eigenvalue problems on the cell describing free periodic vibrations. If the fluctuation shape functions are not derived as solutions to periodic eigenvalue cell problems mentioned above, then the effective moduli (12) of the shell are obtained without specification of the periodic cell problems. This situation is different from that occurring in the known asymptotic homogenization approach, cf., e.g. [20], where only solutions to the periodic cell problems make it possible to define the effective moduli of the structure under consideration.

In the first step of combined modelling, it is assumed that within the asymptotic model, solutions $u_{\alpha}^{0}, w^{0}$ to the problem under consideration are known. Hence, there are also known functions

$$
\begin{aligned}
& u_{0 \alpha}(x, \xi, t)=u_{\alpha}^{0}(x, \xi, t)+h(x) U_{\alpha}(x, \xi, t), \\
& w_{0}(x, \xi, t)=w^{0}(x, \xi, t)+g(x) W(x, \xi, t), \\
& x \in \Omega, \quad(\xi, t) \in \Xi \times \mathrm{I},
\end{aligned}
$$

where $U_{\alpha}, W$ are given by means of (10).

\subsection{Step 2. Tolerance modelling}

The second step of the combined modelling is based on the tolerance modelling technique, $\mathrm{cf}[7,8]$.

The fundamental concepts of the tolerance modelling procedure under consideration are those of two tolerance relations between points and real numbers determined by tolerance parameters, slowly varying functions, tolerance-periodic functions, fluctuation shape functions and the averaging operation.

In what follows, some of the above-mentioned concepts and assumptions will be specified with respect to one-dimensional region $\Omega \equiv\left(0, L_{1}\right)$ defined in this contribution.

Let $F(x)$ be a function defined in $\bar{\Omega}=\left[0, L_{1}\right]$, which is continuous, bounded and differentiable in $\bar{\Omega}$ together with their derivatives up to the $R$ th order. Note that function $F$ can also depend on $\xi \in \bar{\Xi}=\left[0, L_{2}\right]$ and time coordinate $t$ as parameters. Let $\delta \equiv\left(\lambda, \delta_{0}, \delta_{1}, \ldots, \delta_{R}\right)$ be the set of tolerance parameters. The first of them is related to the distances between points in $\Omega$, the second one is related to the distances between values of function $F(\cdot)$ and the $k$ th one to the distances between values of the $k$ th derivative of $F(\cdot), k=1, \ldots, R$. A function $F(\cdot)$ is called slowly varying of the Rth kind with respect to cell $\Delta$ and tolerance parameters $\delta$, $F \in S V_{\delta}^{R}(\Omega, \Delta)$, if and only if the following two conditions are satisfied

$$
\begin{gathered}
\left(\forall(x, y) \in \Omega^{2}\right)\left[(x \stackrel{\lambda}{\approx} y) \Rightarrow F(x) \stackrel{\delta_{0}}{\approx} F(y) \text { and } \partial_{1}^{k} F(x) \stackrel{\delta_{k}}{\approx} \partial_{1}^{k} F(y), \quad k=1,2, \ldots, R\right], \\
(\forall x \in \Omega)\left[\lambda\left|\partial_{1}^{k} F(x)\right| \delta_{k} \approx 0, \quad k=1,2, \ldots, R\right],
\end{gathered}
$$

where symbols " $\stackrel{\lambda}{\approx}$, and " $\approx \delta_{0}, "$, " $\approx$, " denote tolerance relations. 
Roughly speaking, from (15) and (16) it follows that slowly varying function $F(\cdot)$ can be treated as constant on an arbitrary cell and that the products of derivatives of slowly varying function in periodicity direction andmicrostructure length parameter $\lambda$ are treated as negligibly small.

An integrable and bounded function $f(x)$ defined in $\bar{\Omega}=\left[0, L_{1}\right]$, which can also depend on $\xi \in \bar{\Xi}$ and time coordinate $t$ as parameters, is called tolerance-periodic with respect to cell $\Delta$ and tolerance parameters $\delta \equiv\left(\lambda, \delta_{0}\right)$,if for every $x \in \Omega_{\Delta}$ there exist $\Delta$-periodic function $\tilde{f}(\cdot)$ such that $f \mid \Delta(x) \cap \operatorname{Dom} f$ and $\tilde{f} \mid \Delta(x) \cap \operatorname{Dom} \tilde{f}$ are indiscernible in tolerance determined by $\delta \equiv\left(\lambda, \delta_{0}\right)$. Function $\tilde{f}$ is a $\Delta$-periodic approximation of $f$ in $\Delta(x)$. For function $f(\cdot)$ being tolerance-periodic together with its derivatives up to the $R$ th order, we shall write $f \in T P_{\delta}^{R}(\Omega, \Delta), \delta \equiv\left(\lambda, \delta_{0}, \delta_{1}, \ldots, \delta_{R}\right)$.

The concepts of fluctuation shape functions and averaging operation have been explained in Sect. 3.1.

The tolerance modelling is based on two assumptions. The first assumption is called the tolerance averaging approximation. The second one is termed the micro-macro decomposition.

Let $f(\cdot)$ be an integrable periodic function defined in $\bar{\Omega}=\left[0, L_{1}\right]$ and let $F(\cdot) \in S V_{\delta}^{1}(\Omega, \Delta), G(\cdot) \in$ $S V_{\delta}^{2}(\Omega, \Delta), h(\cdot) \in F S^{1}(\Omega, \Delta), g(\cdot) \in F S^{2}(\Omega, \Delta)$. The tolerance averaging approximation has the form

$$
\begin{array}{ll}
<f \partial_{1}^{R} F>(x)=<f>\partial_{1}^{R} F(x)+O(\delta), & R=0,1, \quad \partial_{1}^{0} F \equiv F, \\
<f \partial_{1}^{R} G>(x)=<f>\partial_{1}^{R} G(x)+O(\delta), & R=0,1,2, \quad \partial_{1}^{0} G \equiv G,
\end{array}
$$

and

$$
\begin{aligned}
& <f \partial_{1}(h F)>(x)=<f \partial_{1} h>F(x)+O(\delta), \\
& <f \partial_{1}(g G)>(x)=<f \partial_{1} g>G(x)+O(\delta), \\
& <f \partial_{1}^{2}(g G)>(x)=<f \partial_{1}^{2} g>G(x)+O(\delta),
\end{aligned}
$$

In the course of modelling, terms $O(\delta)$ in (17) and (18) are neglected. Let us observe that the slowly varying functions can be regarded as invariant under averaging.

Approximations given above are applied in the modelling problems discussed in this contribution. For details, the reader is referred to [5-8].

The second fundamental assumption, called the micro-macro decomposition, states that the displacements fields occurring in the starting lagrangian under consideration can be decomposed into unknown averaged (macroscopic) displacements being slowly varying functions in $x \in \Omega$ and highly oscillating fluctuations represented by the known highly oscillating $\lambda$-periodicfluctuation shape functions multiplied by unknownfluctuation amplitudes (microscopic variables) slowly varying in $x$.

In the second step of combined modelling, we introduce the extra micro-macro decomposition superimposed on the known solutions $u_{0 \alpha}, w_{0}$ obtained within the macroscopic model.

$$
\begin{aligned}
& u_{c \alpha}(x, \xi, t)=u_{0 \alpha}(x, \xi, t)+c(x) Q_{\alpha}(x, \xi, t), \\
& w_{b}(x, \xi, t)=w_{0}(x, \xi, t)+b(x) V(x, \xi, t),
\end{aligned}
$$

where fluctuation (microscopic) amplitudes $Q_{\alpha}, V$ are the new slowly varying unknowns, i.e. $Q_{\alpha} \in$ $S V_{\delta}^{1}(\Omega, \Delta), V \in S V_{\delta}^{2}(\Omega, \Delta)$. Functions $c(x) \in F S^{1}(\Omega, \Delta)$ and $b(x) \in F S^{2}(\Omega, \Delta)$ are the new periodic, continuous and highly oscillatingfluctuation shape functions which are assumed to be known in every problem under consideration. These functions have to satisfy conditions: $c \in O(\lambda), \lambda \partial_{1} c \in O(\lambda), b \in O\left(\lambda^{2}\right)$, $\lambda \partial_{1} b \in O\left(\lambda^{2}\right), \lambda^{2} \partial_{11} b \in O\left(\lambda^{2}\right),<\mu c>=<\mu b>=0$, where $\mu(x)$ is the shell mass density.

We substitute the right-hand sides of (19) into (6). The resulting lagrangian is denoted by $L_{c b}$. Then, we average $L_{c b}$ over cell $\Delta$ using averaging formula (8) and applying the tolerance averaging approximation (17), (18). As a result, we obtain function $\left\langle L_{c b}>\right.$ called the tolerance averaging of starting lagrangian (6) in $\Delta$ under superimposed decomposition (19). Next, applying the principle of stationary action, under the extra approximation $1+\lambda / r \approx 1$, we arrive at the system of Euler-Lagrange equations for $Q_{\alpha}, V$, which can be written in an explicit form as

$$
\begin{aligned}
& \leq D^{\alpha 22 \delta}(c)^{2}>\partial_{22} Q_{\delta}-<D^{\alpha 11 \delta}\left(\partial_{1} c\right)^{2}>Q_{\delta}-\leq \mu(c)^{2}>a^{\alpha \beta} \ddot{Q}_{\beta} \\
& \quad=r^{-1}<D^{\alpha 11} \partial_{1} c w_{0}>+<D^{\alpha \beta \gamma 1} \partial_{1} c \partial_{\beta} u_{0 \gamma}>, \\
& <B^{2222}(b)^{2}>\partial_{2222} V+\left[\underline{\left[2<B^{1122} b \partial_{11} b>-4\right.}-\frac{4 B^{1212}\left(\partial_{1} b\right)^{2}>}{{ }^{\alpha \beta 11} \partial_{11} b \partial_{\alpha \beta}} w_{0}>,\right. \\
& +<B^{1111}\left(\partial_{11} b\right)^{2}>V+<\mu(b)^{2}>\ddot{V}=-<B^{\alpha \beta},
\end{aligned}
$$


Equations (20) and (21) together with the micro-macro decomposition (19) constitute the superimposed microscopic model. Coefficients of the derived model equations are constant, and some of them depend on a cell size $\lambda$ (the underlined terms). The right-hand sides of (20) and (21) are known under assumption that $u_{0 \alpha}, w_{0}$ were determined in the first step of modelling. The basic unknowns $Q_{\alpha}, V$ of the model equations must be the slowly varying functions in periodicity directions. This requirement can be verified only a posteriori, and it determines the range of the physical applicability of the model. The boundary conditions for $Q_{\alpha}, V$ should be defined only on boundaries $\xi=0, \xi=L_{2}$.

It can be shown that under assumption that fluctuation shape functions $h(x), g(x)$ of macroscopic model coincide with fluctuation shape functions $c(x), b(x)$ of microscopic model, we can obtain microscopic model equations (20), (21), in which $c(x)$ and $b(x)$ are replaced by $h(x)$ and $g(x)$, respectively, and in which the right-hand sides are equal to zero. Moreover, taking into account a symmetric form of tensor $D^{\alpha \beta \gamma \delta}$ we arrive finally at three equations for unknown fluctuation amplitudes $Q_{1}(x, \xi, t), Q_{2}(x, \xi, t)$ and $V(x, \xi, t)$, which are not conjugated with themselves

$$
\begin{aligned}
& \leq D^{1221}(h)^{2}>\partial_{22} Q_{1}-<D^{1111}\left(\partial_{1} h\right)^{2}>Q_{1}-\leq \mu(h)^{2}>\ddot{Q}_{1}=0, \\
& <D^{2222}(h)^{2}>\partial_{22} Q_{2}-<D^{2112}\left(\partial_{1} h\right)^{2}>Q_{2}-<\mu(h)^{2}>\ddot{Q}_{2}=0, \\
& <B^{2222}(g)^{2}>\partial_{2222} V+\left[2<B^{1122} g \partial_{11} g>-4<B^{1212}\left(\partial_{1} g\right)^{2}>\right] \partial_{22} V \\
& +<B^{1111}\left(\partial_{11} g\right)^{2}>V+<\mu(g)^{2}>\ddot{V}=0 .
\end{aligned}
$$

Equations (22)-(24) are independent of the solutions $u_{0 \alpha}, w_{0}$ obtained in the framework of the macroscopic model. Hence, they describe selected problems of the shell micro-dynamics (e.g. the free micro-vibrations, propagation of waves related to the micro-fluctuation amplitudes) independently of the shell macro-dynamics. Moreover, micro-dynamic behaviour of the shell in the axial and circumferential directions can be analysed independently of its micro-dynamic behaviour in the direction normal to the shell midsurface.

Microscopic model equations (22)-(24) also describe certain time-boundary and space-boundary phenomena strictly related to the specific form of initial and boundary conditions imposed on unknown fluctuation amplitudes $Q_{\alpha}, V$. That is why, these equations are referred to as the boundary layer equations, where the term "boundary" is related both to time and space.

Since equations (22)-(24) are not conjugated with themselves, the micro-dynamic behaviour of the shells in the axial, circumferential and normal directions can be investigated independently of each other.

\subsection{Combined asymptotic-tolerance model}

Summarizing results obtained in Sects. 3.1 and 3.2, we conclude that the combined asymptotic-tolerance model of selected dynamic problems for the uniperiodic shells under consideration presented here following Tomczyk [8] is represented by:

(a) Macroscopic model defined by Eq. (11) for $u_{\alpha}^{0}, w^{0}$ with expressions (10) for $U_{\alpha}, W$, formulated by means of the consistent asymptotic modelling and being independent of the microstructure length. Unknowns of this model must be continuous and bounded functions in $x$. It is assumed that in the framework of this model, the solutions (14) to the problem under consideration are known.

(b) Superimposed microscopic model equations (20), (21) derived by means of the tolerance (non-asymptotic) modelling and having constant coefficients depending also on a cell size $\lambda$ (underlined terms). Microscopic model equations (20), (21) are coupled with the macroscopic model equations (11) by means of the known solutions (14) obtained in the framework of the asymptotic model. Unknown fluctuation amplitudes $Q_{\alpha}, V$ of the tolerance model must be slowly varying functions in $x$.

(c) Decomposition

$$
\begin{aligned}
& u_{\alpha}(x, \xi, t)=u_{\alpha}^{0}(x, \xi, t)+h(x) U_{\alpha}(x, \xi, t)+c(x) Q_{\alpha}(x, \xi, t), \\
& w(x, \xi, t)=w^{0}(x, \xi, t)+g(x) W(x, \xi, t)+b(x) V(x, \xi, t), \\
& x \in \Omega, \quad(\xi, t) \in \Xi \times \mathrm{I},
\end{aligned}
$$

where functions $u_{\alpha}^{0}, U_{\alpha}, w^{0}, W$ have to be obtained in the first step of combined modelling, i.e. in the framework of the consistent asymptotic modelling. 
Coefficients of all equations derived in the framework of combined modelling are constant in contrast to coefficients in starting Eq. (7) which are discontinuous, highly oscillating and periodic in $x$. Moreover, some of them depend on a cell size $\lambda$. Thus, the combined model can be applied to the analysis of many phenomena caused by the length-scale effect.

Under special conditions imposed on the fluctuation shape functions, we can obtain microscopic model equations (22)-(24), which are independent of the solutions obtained in the framework of the macroscopic model. It means that an important advantage of the combined model is that it makes it possible to separate the macroscopic description of some special dynamic problems from the microscopic description of these problems.

For details, the reader is referred to Tomczyk [8].

It should be noted that the combined asymptotic-tolerance model of dynamic problems for cylindrical shells with periodic structure in both circumferential and axial directions (biperiodic shells) proposed in Tomczyk and Litawska [11] cannot be applied for analysis of dynamic problems for uniperiodic shells considered here. Model presented in [11] is derived in the framework of the extended version of the tolerance modelling technique based on a new notion of weakly slowly varying function, cf. Tomczyk and Woźniak [12]. For this function, restrictive condition (16) and approximations (18) do not hold. Moreover, in the non-asymptotic-tolerance approach, the uniperiodic shells are not special cases of shells with two-directional periodic structure.

It should be also noted that the combined asymptotic-tolerance models for functionally graded cylindrical shells are presented by Tomczyk and Szczerba in $[17,18]$. Coefficients of governing equations of these models are not constant. They are smooth and slowly varying either in circumferential direction [17] or in the axial one [18].

Some applications of micro-dynamic Eqs. (22)-(24) will be shown in the next section.

\section{Examples of applications}

In this section, we shall investigate two special micro-dynamic problems applying Eqs. (22)-(24). The first of them deals with free cell-depending micro-vibrations. The second one deals with propagation of the waves related to micro-fluctuations of axial displacements.

It has to be emphasized that these aforementioned special micro-dynamic problems can be studied in the framework of neither the asymptotic models nor the known commercial numerical models for the periodic shells under consideration.

\subsection{Formulation of the problem}

The object of considerations is a thin cylindrical shell with $L_{1}, L_{2}, r, d$ as its circumferential length, axial length, midsurface curvature radius and constant thickness, respectively. The shell has a periodically heterogeneous structure along circumferential direction and constant structure in the axial direction. It is assumed that the shell is made of two homogeneous elastic isotropic materials, which are perfectly bonded on interfaces, cf. Fig. 2. The free micro-vibration problem will be studied for an open simply supported shell, i.e. for a shell with hinged edges and with supports free to move, cf. [19]. The wave propagation problem will be investigated for a closed shell (obviously, in this case $L_{1}=2 \pi r$ ). Moreover, we assume that $L_{2} \geq L_{1}$.

The shell's mass density per midsurface unit area $\mu(x)$ and stiffness tensors $D^{\alpha \beta \gamma \delta}(x), B^{\alpha \beta \gamma \delta}(x)$ are described by functions $\lambda$-periodic in $x$ and independent of $\xi$.

The basic cell $\Delta$ is defined by: $\Delta \equiv[-\lambda / 2, \lambda / 2]$, where $\lambda$ is a cell length dimension in $x \equiv x^{1}$ direction, cf. Figs. 2 and 3. We recall that the microstructure length parameter $\lambda$ has to satisfy conditions: $\lambda / d>>1, \quad \lambda / r<<1$ and $\lambda / L_{1}<<1$. Setting $z \equiv z^{1} \in[-\lambda / 2, \lambda / 2]$, we assume that the cell has a symmetry axis for $z=0$. Inside the cell, the geometrical, elastic and inertial properties of the shell are described by symmetric (i.e. even) functions of argument $z$.

Properties of the component materials are described by: Young's moduli $E_{1}, E_{2}$, Poisson's ratios $v_{1}, v_{2}$ and mass densities $\rho_{1}, \rho_{2}$, cf. Fig. 3. It is assumed that elastic $E(\cdot)$ and inertial $\rho(\cdot)$ properties of the composite shell are periodic functions in $x$, but Poisson's ratio $v \equiv v_{1}=v_{2}$ is constant. Inside the cell, functions $E(z)$, $\rho(z)$ take the form

$$
E(z), \rho(z)=\left\{\begin{array}{l}
E_{1}, \rho_{1} \text { for } \quad z \in(-\eta \lambda / 2, \eta \lambda / 2) \\
E_{2}, \rho_{2} \text { for } \quad z=[-\lambda / 2,-\eta \lambda / 2] \cup[\eta \lambda / 2, \lambda / 2]
\end{array}\right.
$$




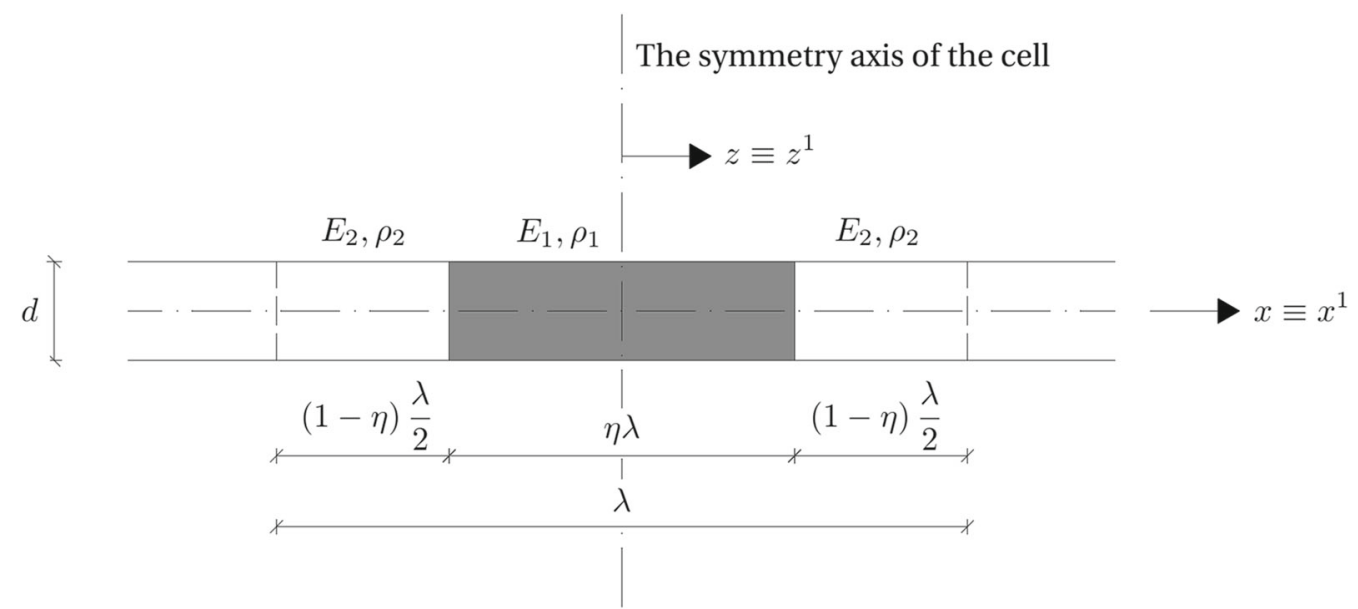

Fig. 3 Basic cell $\Delta \equiv[-\lambda / 2, \lambda / 2]$ of the uniperiodic shell

where $\eta \in[0,1]$ is a parameter describing distribution of material properties in the cell, cf. Fig. 3 .

Inside the cell, the rigidities $D^{\alpha \beta \gamma \delta}(z), B^{\alpha \beta \gamma \delta}(z), z \in \Delta$, of the shell are described by: $D^{\alpha \beta \gamma \delta}(z)=$ $D(z) H^{\alpha \beta \gamma \delta}, B^{\alpha \beta \gamma \delta}(z)=B(z) H^{\alpha \beta \gamma \delta}$, where $D(z)=E(z) d /\left(1-v^{2}\right), B(z)=E(z) d^{3} /\left(12\left(1-v^{2}\right)\right)$ and the nonzero components of tensor $H^{\alpha \beta \gamma \delta}$ are: $H^{1111}=H^{2222}=1, H^{1122}=H^{2211}=v, H^{1212}=H^{1221}=$ $H^{2121}=H^{2112}=(1-v) / 2$. The shell mass density per midsurface unit area is given by $\mu(z)=\rho(z) d$.

The fluctuation shape functions $h(z) \in F S^{1}(\Omega, \Delta), g(z) \in F S^{2}(\Omega, \Delta)$ describe the expected form of displacement disturbances caused by a periodic structure of the shell. It means that they should approximate the expected principal modes of the shell's free vibrations. These modes have to be $\lambda$-periodic, and their mean values in every cell must be equal to zero. On the basis of knowledge of the physically reasonable approximations of principal modes of free vibrations in thin Kirchhoff-Love-type periodic cylindrical shells, cf. Tomczyk [8], and also in thin Kirchhoff-type periodic plates, cf. Jędrysiak [22], in the problem under consideration the fluctuation shape functions can be taken as: $h(z)=\lambda \sin (2 \pi z / \lambda), g(z)=\lambda^{2}[\cos (2 \pi z / \lambda)+\widehat{c}], z \in \Delta(x), x \in \Omega$, where constant $\widehat{c}$, calculated from condition $<\mu g\rangle=0$, is equal to $\widehat{c}=-\left(\rho_{1}-\rho_{2}\right) \sin (\eta \pi)\left[\pi\left(\eta \rho_{1}+(1-\eta) \rho_{2}\right)\right]^{-1}$.

The subsequent analysis will be based on Eqs. (22)-(24) describing the shell micro-dynamics.

\subsection{Micro-vibrations}

The cell-depending free micro-vibration frequencies of an open simply supported cylindrical shell described in the previous subsection will be determined and discussed. Micro-dynamic equations (22)-(24) will be applied.

Solutions to Eqs. (22)-(24) satisfying boundary conditions for the shell simply supported on edges $x=0$, $x=L_{1}, \xi=0, \xi=L_{2}$, i.e. conditions [19]

$$
\begin{aligned}
& \partial_{1} Q_{1}(x=0, \xi, t)=Q_{2}(x=0, \xi, t)=V(x=0, \xi, t)=\partial_{11} V(x=0, \xi, t)=0, \\
& \partial_{1} Q_{1}\left(x=L_{1}, \xi, t\right)=Q_{2}\left(x=L_{1}, \xi, t\right)=V\left(x=L_{1}, \xi, t\right)=\partial_{11} V\left(x=L_{1}, \xi, t\right)=0, \\
& Q_{1}(x, \xi=0, t)=\partial_{2} Q_{2}(x, \xi=0, t)=V(x, \xi=0, t)=\partial_{22} V(x, \xi=0, t)=0, \\
& Q_{1}\left(x, \xi=L_{2}, t\right)=\partial_{2} Q_{2}\left(x, \xi=L_{2}, t\right)=V\left(x, \xi=L_{2}, t\right)=\partial_{22} V\left(x, \xi=L_{2}, t\right)=0,
\end{aligned}
$$

can be assumed in the form

$$
\begin{aligned}
& Q_{1}(x, \xi, t)=A \cos \left(\pi x / L_{1}\right) \sin \left(\pi \xi / L_{2}\right) \cos (\bar{\omega} t), \\
& Q_{2}(x, \xi, t)=B \sin \left(\pi x / L_{1}\right) \cos \left(\pi \xi / L_{2}\right) \cos (\breve{\omega} t), \\
& V(x, \xi, t)=C \sin \left(\pi x / L_{1}\right) \sin \left(\pi \xi / L_{2}\right) \cos (\omega t),
\end{aligned}
$$

where $A \neq 0, B \neq 0, C \neq 0$ are micro-vibration amplitudes being arbitrary constants, $\pi / L_{1}$ and $\pi / L_{2}$ are wave numbers and $\bar{\omega}, \breve{\omega}, \omega$ are frequencies of free micro-vibrations along $x$-and $\xi$-coordinates and in direction normal to the shell midsurface, respectively. 
Substituting the right-hand sides of (27), (28) and (29) into Eqs. (22), (23) and (24), respectively, and setting $\bar{h}(z)=\lambda^{-1} h(z), \breve{g}(z)=\lambda^{-1} g(z), \bar{g}(z)=\lambda^{-2} g(z)$, under extra denotations

$$
\begin{aligned}
& \bar{d} \equiv<D^{1111}\left(\partial_{1} h\right)^{2}>, \quad \bar{e} \equiv<D^{1212}\left(\partial_{1} h\right)^{2}>, \\
& \bar{k} \equiv \pi^{2}\left(L_{2}\right)^{-2}<D^{1212}(\bar{h})^{2}>, \\
& \bar{l} \equiv \pi^{2}\left(L_{2}\right)^{-2}<D^{2222}(\bar{h})^{2}>, \\
& \tilde{a} \equiv 2 \pi^{2}\left(L_{2}\right)^{-2}\left(<B^{1122} \bar{g} \partial_{11} g>-2<B^{1212}\left(\partial_{1} \tilde{g}\right)^{2}>\right), \\
& \tilde{d} \equiv<B^{1111}\left(\partial_{11} g\right)^{2}>, \\
& \tilde{e} \equiv \pi^{4}\left(L_{2}\right)^{-4}<B^{2222}(\bar{g})^{2}>, \\
& \bar{\mu} \equiv<\mu(\bar{h})^{2}>, \quad \tilde{\mu} \equiv<\mu(\bar{g})^{2}>,
\end{aligned}
$$

we arrive at the following formulae for

- free micro-vibration frequency $\bar{\omega}$ in circumferential direction

$$
(\bar{\omega})^{2}=\frac{\bar{k}}{\bar{\mu}}+\frac{\bar{d}}{\lambda^{2} \bar{\mu}},
$$

- free micro-vibration frequency $\breve{\omega}$ in axial direction

$$
(\breve{\omega})^{2}=\frac{\bar{l}}{\bar{\mu}}+\frac{\bar{e}}{\lambda^{2} \bar{\mu}} .
$$

- transversal free micro-vibration frequency $\omega$

$$
\omega^{2}=\frac{\tilde{e}}{\tilde{\mu}}-\frac{\tilde{a}}{\lambda^{2} \tilde{\mu}}+\frac{\tilde{d}}{\lambda^{4} \tilde{\mu}} .
$$

The free micro-vibration frequencies given by (31)-(33) depend on microstructure length parameter $\lambda$.

\subsubsection{Numerical results}

Let us define the following dimensionless free micro-vibration frequencies

$$
\begin{aligned}
& (\bar{\Omega})^{2} \equiv\left(1-v^{2}\right) \rho_{1}\left(L_{1}\right)^{2}\left(E_{1}\right)^{-1}(\bar{\omega})^{2}, \\
& (\widetilde{\Omega})^{2} \equiv\left(1-v^{2}\right) \rho_{1}\left(L_{1}\right)^{2}\left(E_{1}\right)^{-1}(\breve{\omega})^{2}, \\
& \Omega^{2} \equiv\left(1-v^{2}\right) \rho_{1}\left(L_{1}\right)^{2}\left(E_{1}\right)^{-1}(\omega)^{2},
\end{aligned}
$$

where frequencies $\bar{\omega}, \breve{\omega}, \omega$ are determined by formulae (31)-(33), respectively.

The subsequent calculations will be made for $\eta=0.5$, Poisson's ratio $v=0.3$, for fixed ratios $L_{2} / L_{1}=2$, $d / \lambda=0.1$ and for various ratios $\varepsilon \equiv \lambda / L_{1} \in[0.05,0.1], \kappa \equiv E_{2} / E_{1} \in[0.01,1], \phi \equiv \rho_{2} / \rho_{1} \in[0.01,1]$.

In Figs. 4, 6 and 8, there are presented diagrams of dimensionless free micro-vibration frequencies given by (34) versus dimensionless microstructure length parameter $\varepsilon \equiv \lambda / L_{1} \in[0.05,0.1]$. These diagrams are made for three pairs of ratios: $(\kappa=0.9, \phi=0.1),(\kappa=0.5, \phi=0.5),(\kappa=0.1, \phi=0.9)$.

In Figs. 5a, 7a, 9a, the diagrams of dimensionless frequencies (34) versus ratio $K \equiv E_{2} / E_{1} \in[0.01,1]$ are presented. These plots are made for $\phi=0.1, \phi=0.5, \phi=0.9$ and for $\varepsilon=0.1$.

In Figs. 5b, 7b, 9b, the plots of dimensionless frequencies (34) versus ratio $\phi \equiv \rho_{2} / \rho_{1} \in[0.01,1]$ are shown. These plots are made for $\kappa=0.1, \kappa=0.5, \kappa=0.9$ and for $\varepsilon=0.1$.

\subsubsection{Discussion of computational results}

On the basis of results shown in Figs. 4, 5, 6, 7, 8 and 9, the following conclusions can be formulated:

1. Values of the dimensionless frequencies decrease with the increasing of ratio $\lambda / L_{1}$, i.e. with the decreasing of differences between period length $\lambda$ and the length dimension $L_{1}$ of the shell midsurface in periodicity direction, cf. Figs. 4, 6, 8. 


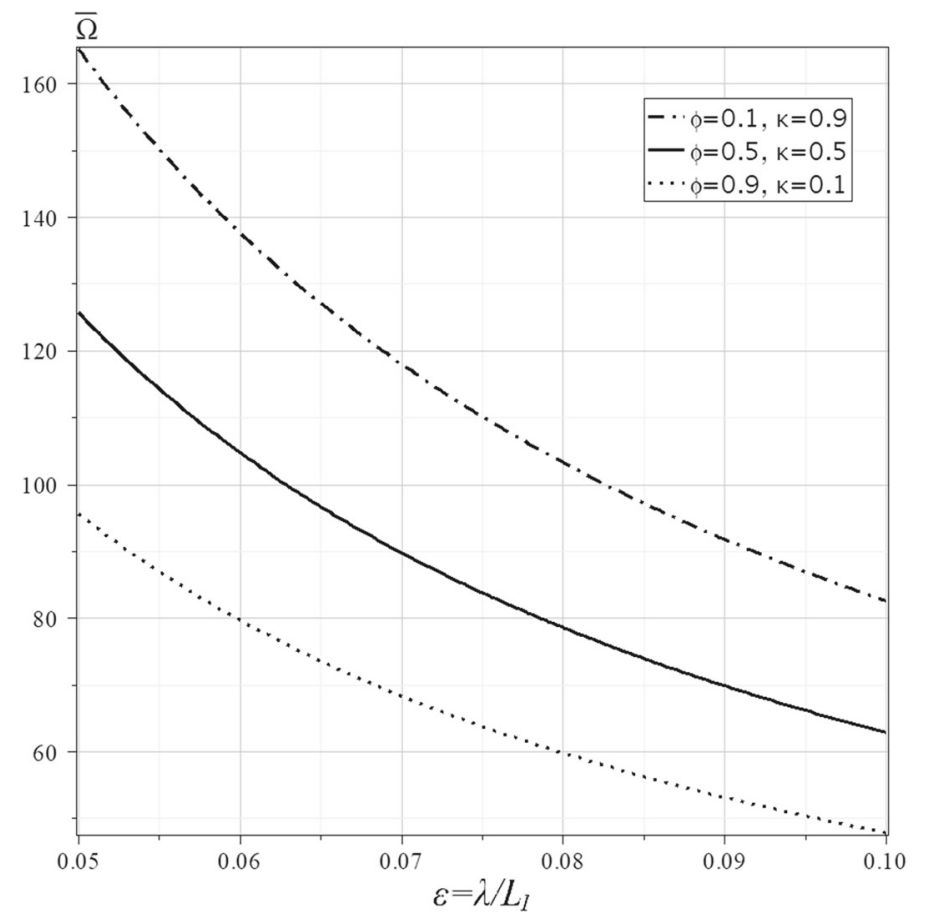

Fig. 4 Diagrams of dimensionless frequencies $\bar{\Omega}$ of free micro-vibrations in circumferential direction versus dimensionless microstructure length parameter $\varepsilon \equiv \lambda / L_{1} ; \phi \equiv \rho_{2} / \rho_{1}, \kappa \equiv E_{2} / E_{1}$

(a)

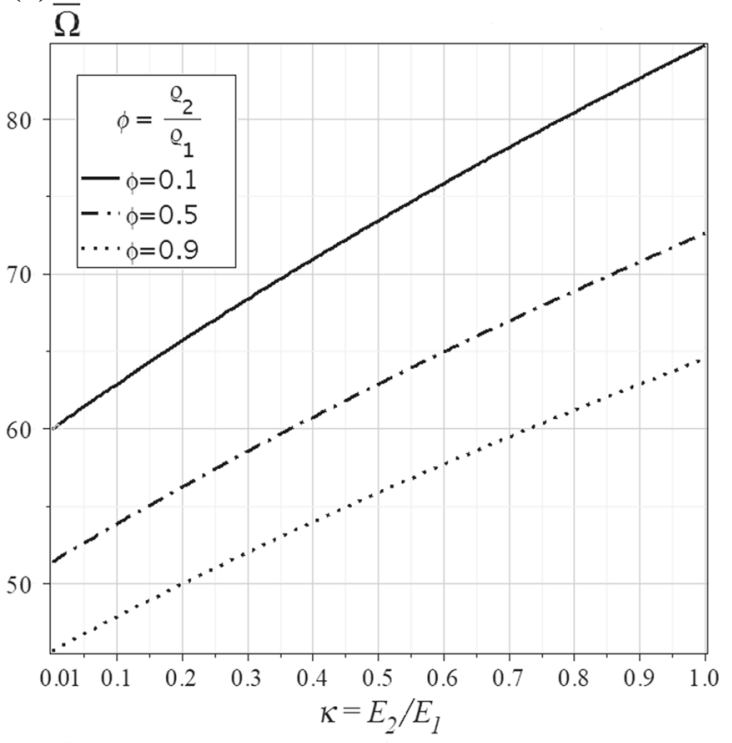

(b)

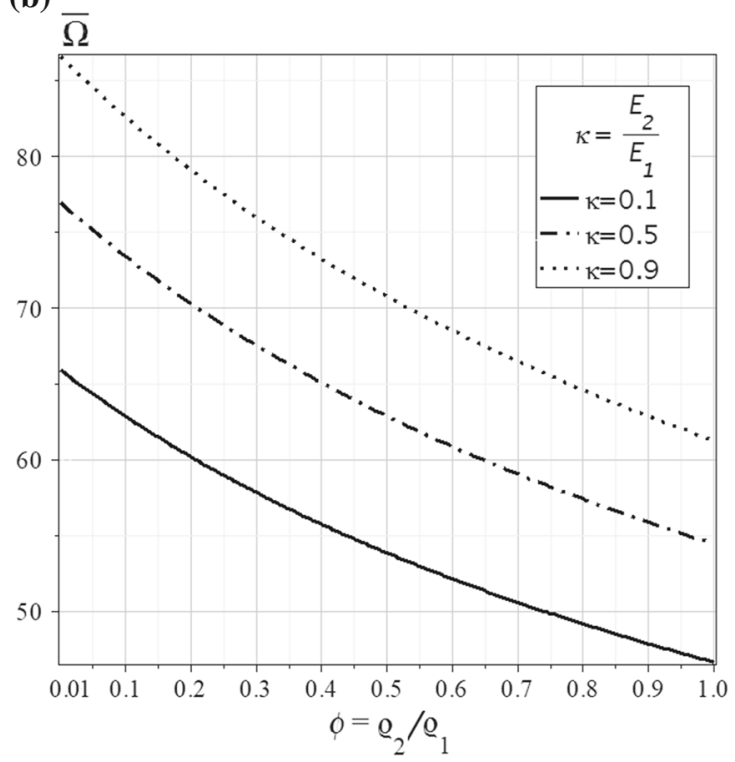

Fig. 5 Diagrams of dimensionless frequencies $\bar{\Omega}$ of free micro-vibrations in circumferential direction versus: a ratio $E_{2} / E_{1}$ and b ratio $\rho_{2} / \rho_{1} ; \lambda / L_{1}=0.1$

2. Values of dimensionless free micro-vibration frequencies increase with the increasing of ratio $E_{2} / E_{1} \in$ $[0.01,1]$, i.e. with the decreasing of differences between elastic properties of the shell component materials, cf. Figs. 5a, 7a, 9a, but they decrease with the increasing of ratio $\rho_{2} / \rho_{1} \in[0.01,1]$, i.e. with the decreasing of differences between inertial properties of the component materials, cf. Figs. 5b, 7b, $9 \mathrm{~b}$.

3. The highest values of frequencies $\bar{\Omega}$, cf. Fig. $5, \widetilde{\Omega}$, cf. Fig. 7, $\Omega$, cf. Fig. 9 , are obtained for pair of ratios $\left(E_{2} / E_{1}=1, \rho_{2} / \rho_{1}=0.01\right)$, i.e. for a periodic shell with a very strong inertial heterogeneity and with 


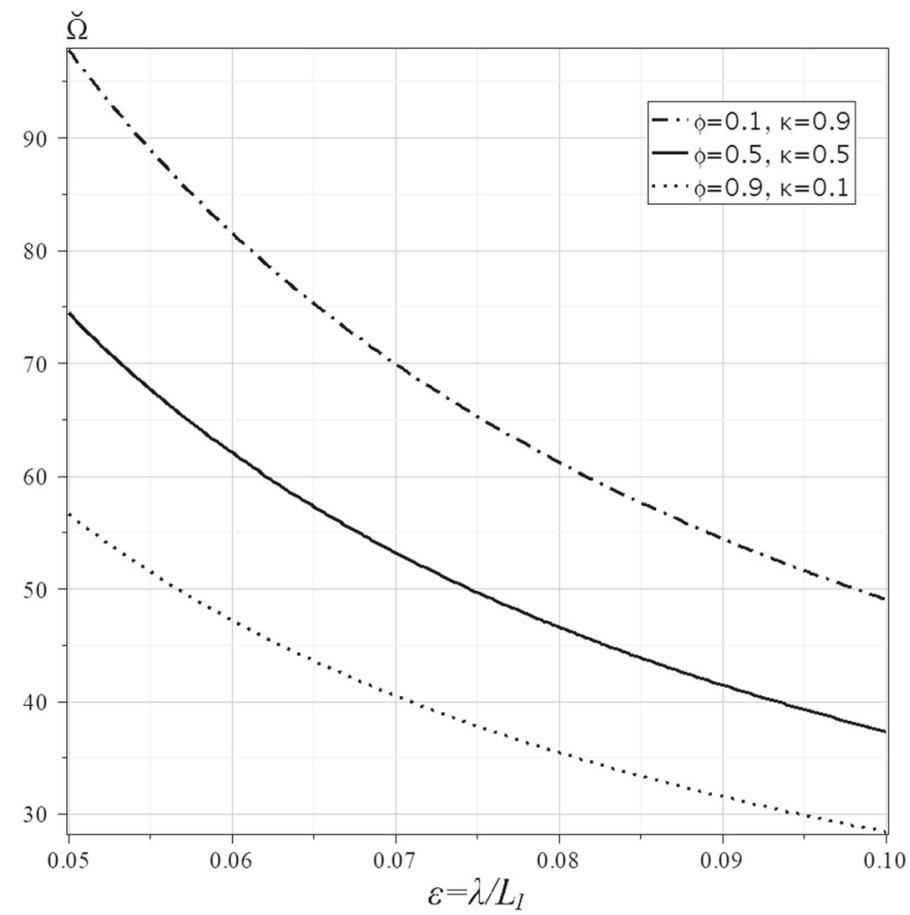

Fig. 6 Diagrams of dimensionless frequencies $\breve{\Omega}$ of free micro-vibrations in axial direction versus dimensionless microstructure length parameter $\varepsilon \equiv \lambda / L_{1} ; \phi \equiv \rho_{2} / \rho_{1}, \kappa \equiv E_{2} / E_{1}$

(a)

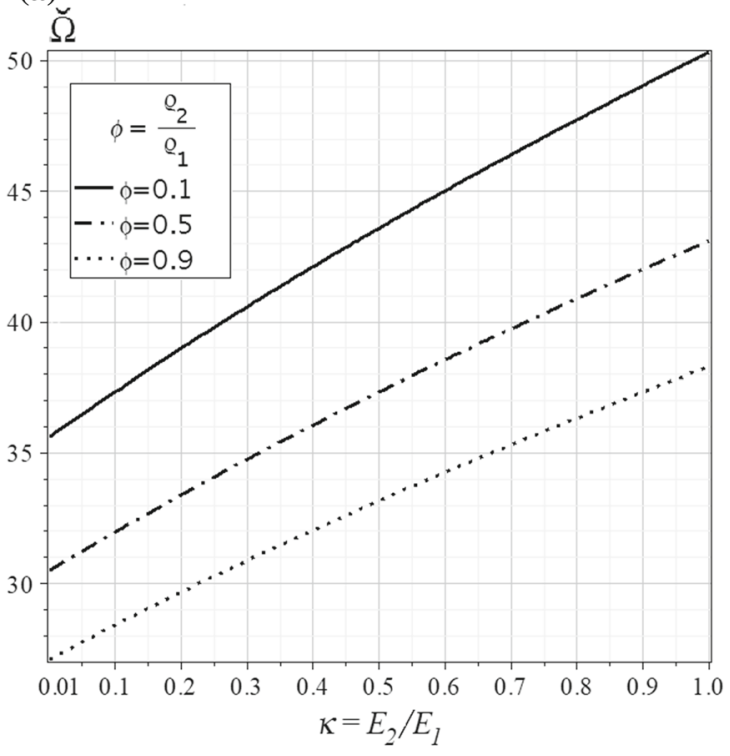

(b)

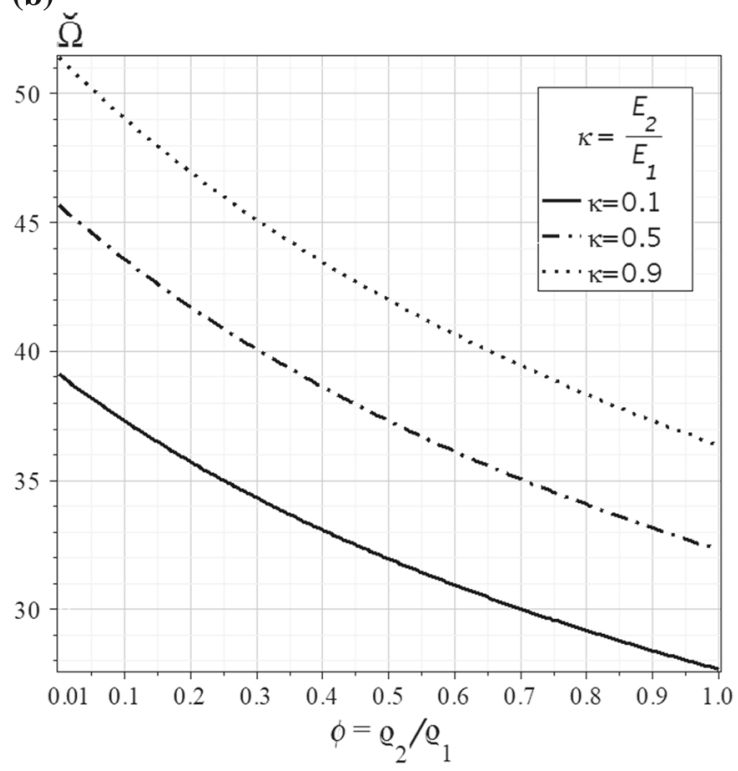

Fig. 7 Diagrams of dimensionless frequencies $\breve{\Omega}$ of free micro-vibrations in axial direction versus: a ratio $E_{2} / E_{1}$ and $\mathbf{b}$ ratio $\rho_{2} / \rho_{1} ; \lambda / L_{1}=0.1$

elastic homogeneous structure. The smallest values of these frequencies are obtained for pair of ratios $\left(E_{2} / E_{1}=0.01, \rho_{2} / \rho_{1}=1\right)$, i.e. for a periodic shell with a very strong elastic heterogeneity and with inertial homogeneous structure. 


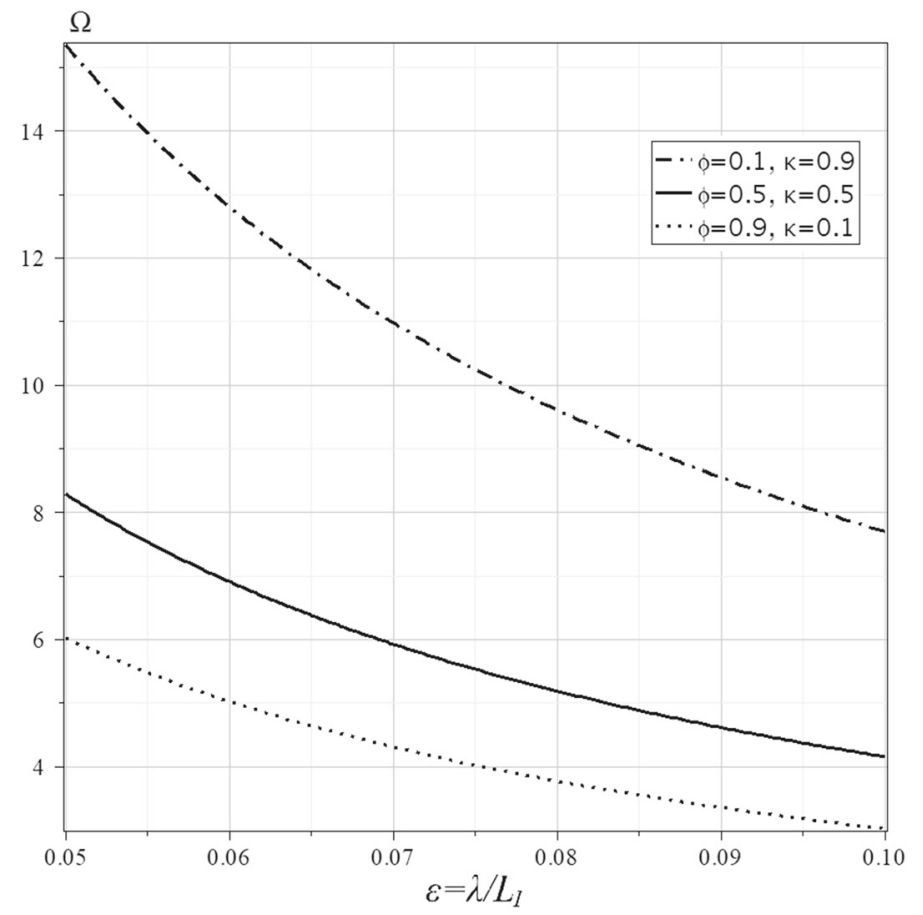

Fig. 8 Diagrams of dimensionless frequencies $\Omega$ of transversal free micro-vibrations versus dimensionless microstructure length parameter $\varepsilon \equiv \lambda / L_{1} ; \phi \equiv \rho_{2} / \rho_{1}, \kappa \equiv E_{2} / E_{1}$

(a)

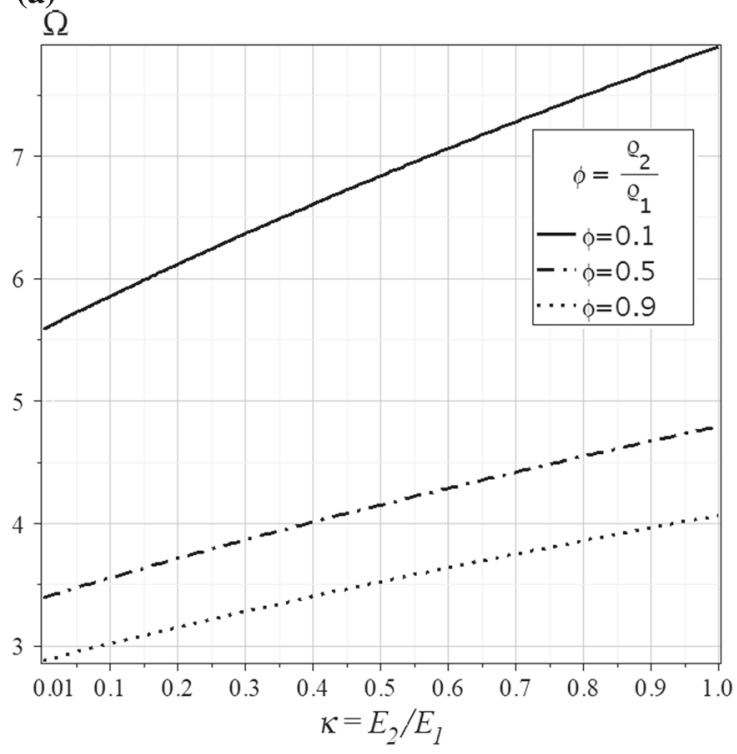

(b)

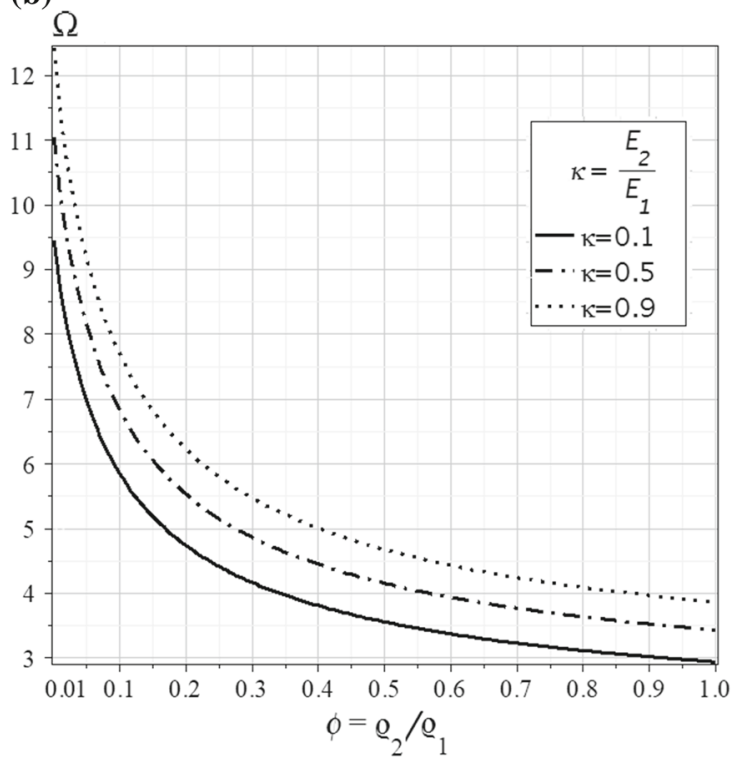

Fig. 9 Diagrams of dimensionless frequencies $\Omega$ of transversal free micro-vibrations versus: a ratio $E_{2} / E_{1}$ and $\mathbf{b}$ ratio $\rho_{2} / \rho_{1}$; $\lambda / L_{1}=0.1$

\subsection{Long wave propagation problem}

Now, let the shell under consideration be closed and unbounded along the axial coordinate $\xi$. We shall analyse the long wave propagation problem. We deal with long waves if condition $\lambda / L<<1$ holds, where $\lambda$ is the characteristic length dimension of the cell and $L$ is the wavelength. The waves related to micro-fluctuation amplitude $Q_{2}$ are taken into account. Hence, Eq. (23) describing the shells' micro-dynamics in an axial direction 
will be applied. Let the investigated problem be rotationally symmetric with a period $\lambda / r$; thus, unknown $Q_{2}(\cdot, t)$ of (23) is independent of $x$. We look for solution to Eq. (23) in the form $Q_{2}(\xi, t)=F(\xi-c t)$, where $c$ is the wave propagation velocity. Setting $\bar{h}=\lambda^{-1} h$, from Eq. (23) we obtain

$$
\left(c^{2}-\tilde{c}^{2}\right) \partial_{22} F+\bar{c}^{2} \lambda^{-2} F=0,
$$

where speeds $\tilde{c}$ and $\bar{c}$ are defined by

$$
\tilde{c}^{2} \equiv \frac{<D^{2222}(\bar{h})^{2}>}{<\mu(\bar{h})^{2}>}, \quad \bar{c}^{2} \equiv \frac{<D^{2112}\left(\partial_{1} h\right)^{2}>+<D^{2222}\left(\partial_{2} h\right)^{2}>}{<\mu(\bar{h})^{2}>} .
$$

Equation (35) implies the following special cases of wave propagation in the uniperiodic shells under consideration

(a) sinusoidal waves if $c>\tilde{c}$,

(b) exponential waves if $c<\tilde{c}$,

(c) degenerate case if $c=\tilde{c}$

The above effect cannot be analysed in the framework of asymptotic models.

In order to determine the dispersion relation for the case (a), let us substitute to Eq. (23) solution of the form $Q_{2}(\xi, t)=A \sin (k(\xi-c t)), k=2 \pi / L$, where $L \equiv L_{2}$ and $k$ are the wavelength and the wave number, respectively; $A$ is an arbitrary constant. It is assumed that $L_{2}>L_{1}=2 \pi r$, and hence $L>>\lambda$. The nontrivial solution $(A \neq 0)$ exists only if

$$
\left[(k \lambda)^{2} c^{2}-(k \lambda)^{2} \tilde{c}^{2}-\bar{c}^{2}\right]=0,
$$

where under assumption that $L>>\lambda$ the following condition holds $k \lambda=2 \pi \lambda / L<<1$.

The above equation describes the effect of dispersion. It can be seen that for $k \lambda \rightarrow 0$, the dispersion effect disappears. From Eq. (37), it follows that the dispersive long waves related to micro-fluctuation amplitude $Q_{2}(\xi, t)$ can propagate across the unbounded uniperiodic shells under consideration with propagation speed

$$
c^{2}=\tilde{c}^{2}+\bar{c}^{2}(k \lambda)^{-2}
$$

depending on microstructure size $\lambda$. Note that for homogeneous isotropic shells, expression (38) leads to the well-known results $c^{2}=D / \mu, D=E \delta /\left(1-v^{2}\right)$, where $E, v, \delta, \mu$ are Young's modulus, Poisson's ratio, the shell thickness and mass density of the shell material, respectively, cf. Kaliski [19].

\subsubsection{Numerical results}

Let us define the following dimensionless wave propagation speed

$$
C^{2} \equiv \rho_{1}\left(E_{1}\right)^{-1} c^{2}
$$

where speed $c$ is determined by formula (38).

The subsequent calculations will be made for parameter $\eta=0.05,0.25,0.5$, where $\eta$ is a parameter describing distribution of material properties in the cell, for Poisson's ratio $v=0.3$, for fixed ratio $d / \lambda=0.1$ and for various ratios $\varepsilon \equiv \lambda / L \in[0.0001,0.01], \kappa \equiv E_{2} / E_{1} \in[0.01,1], \phi \equiv \rho_{2} / \rho_{1} \in[0.01,1]$.

In Fig. 10, the diagrams of dimensionless wave propagation speed given by (39) versus dimensionless microstructure length parameter $\varepsilon \equiv \lambda / L \in[0.0001,0.01]$ are presented. These diagrams are made for pair of ratios: $(\kappa=0.01, \phi=0.01)$ and for $\eta=0.05,0.25,0.5$.

In Fig. 11, the diagrams of dimensionless speed (39) versus ratio $K \equiv E_{2} / E_{1} \in[0.01,1]$ are presented. These plots are made for $\varepsilon=0.01, \eta=0.25$ and $\phi=0.01,0.1,0.5,0.9,1$.

In Fig. 12, the plots of dimensionless speed (39) versus ratio $\phi \equiv \rho_{2} / \rho_{1} \in[0.01,1]$ are shown. These plots are made for $\varepsilon=0.01, \eta=0.25$ and $\kappa=0.01,0.1,0.5,0.9,1$. 


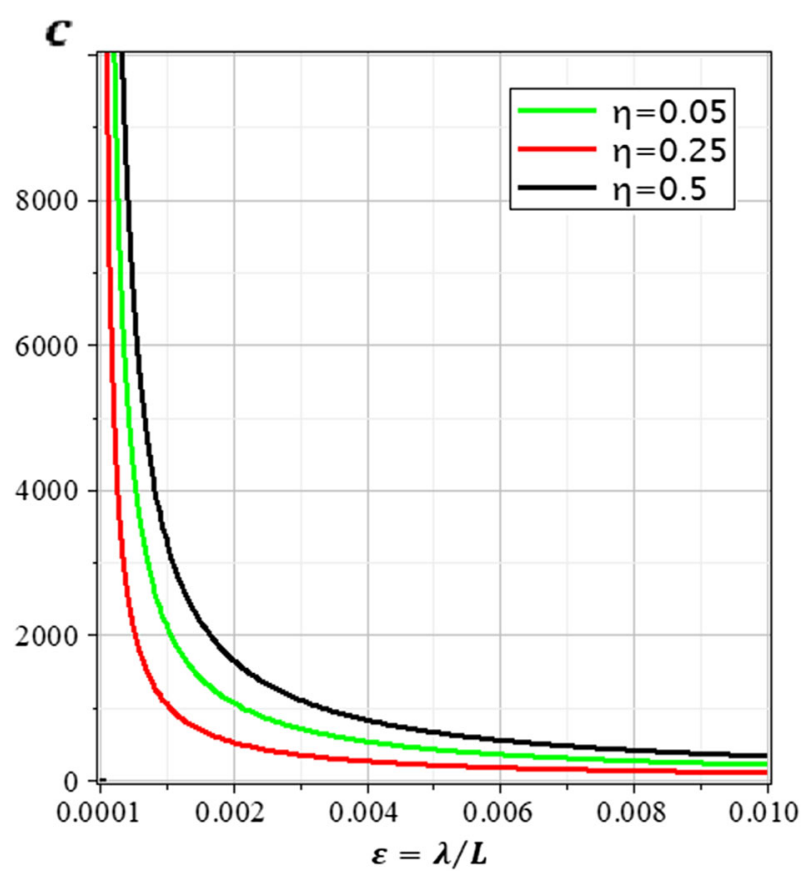

Fig. 10 Diagrams of dimensionless wave propagation speed $C$ versus dimensionless microstructure length parameter $\varepsilon \equiv \lambda / L$; $E_{2} / E_{1}=0.01, \rho_{2} / \rho_{1}=0.01$

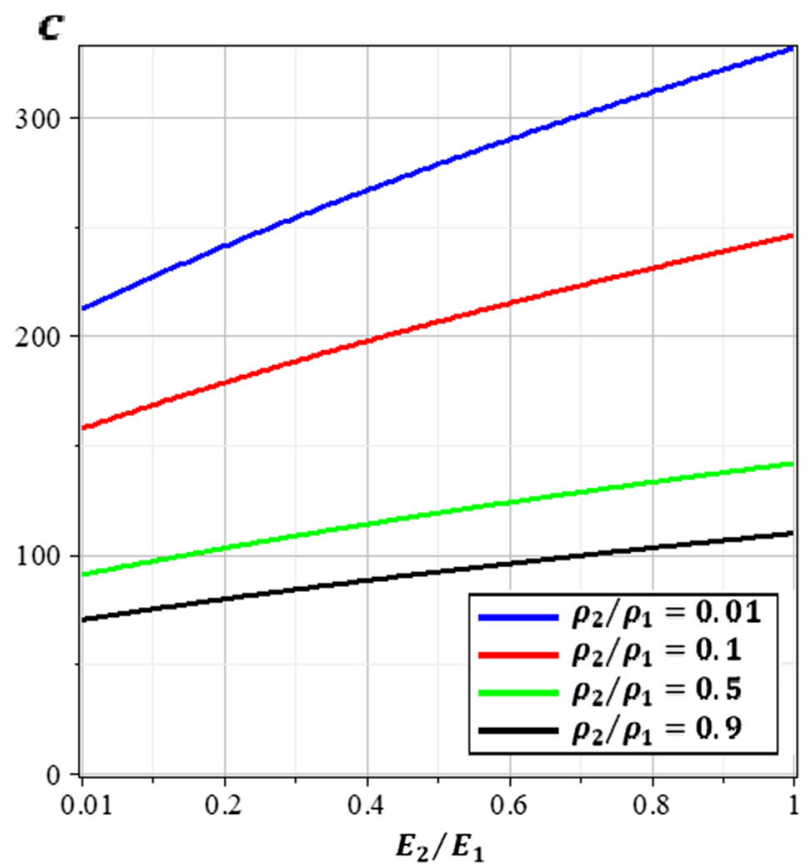

Fig. 11 Diagrams of dimensionless wave propagation speed $C$ versus ratio $E_{2} / E_{1} ; \lambda / L=0.01, \eta=0.25$

\subsubsection{Discussion of analytical and computational results}

It was shown that the tolerance-periodic heterogeneity of the shells leads to exponential waves and to dispersion effects, which cannot be analysed in the framework of the asymptotic models for periodic shells. Moreover, the new wave propagation speed depending on the microstructure size has been obtained, cf. formula (38).

On the basis of results shown in Figs. 10, 11 and 12, the following conclusions can be formulated: 


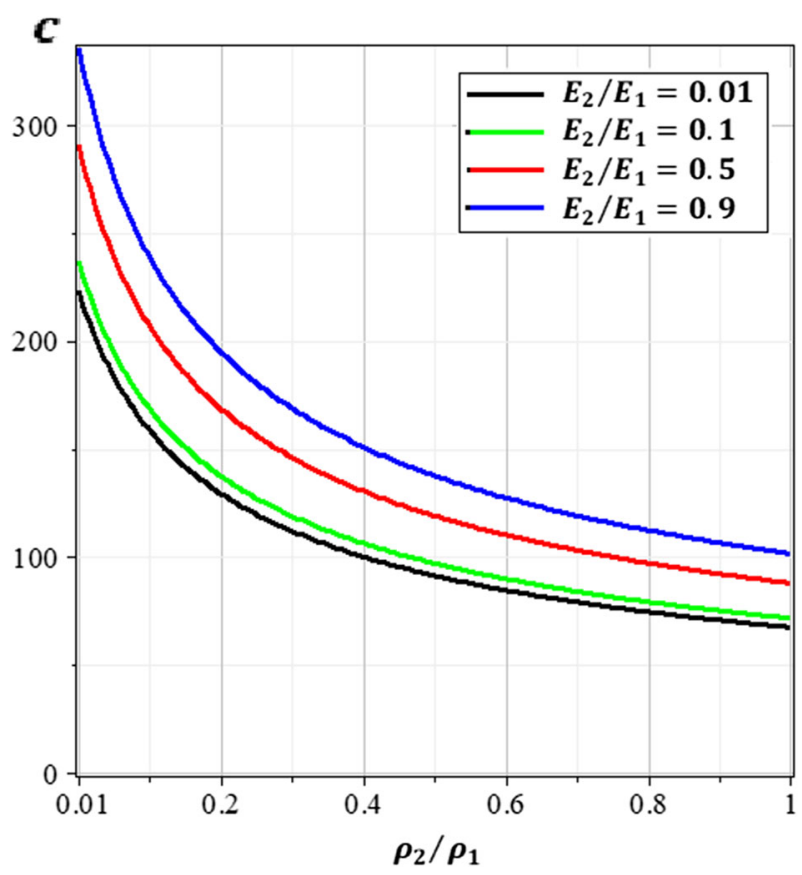

Fig. 12 Diagrams of dimensionless wave propagation speed $C$ versus ratio $\rho_{2} / \rho_{1} ; \lambda / L=0.01, \eta=0.25$

1. Values of the dimensionless wave propagation velocity $C$ decrease with the increasing of ratio $\lambda / L$, i.e. with the decreasing of differences between period length $\lambda$ and the wavelength $L \equiv L_{2}$, cf. Fig. 10. The strongest decrease in the dimensionless speed $C$ takes place for $\varepsilon \equiv \lambda / L \in[0.0001,0.001]$.

2. Values of dimensionless speed $C$ increase with the decrease in parameter $\eta$ describing distribution of material properties in the cell, i.e. with the decrease in the share of stronger material in the cell., cf. Fig. 10.

3. Values of dimensionless wave propagation velocity increase with the increasing of ratio $E_{2} / E_{1} \in[0.01,1]$, i.e. with the decreasing of differences between elastic properties of the shell component materials, cf. Fig. 11, but they decrease with the increasing of ratio $\rho_{2} / \rho_{1} \in[0.01,1]$, i.e. with the decreasing of differences between inertial properties of the component materials, cf. Fig. 12.

4. The highest values of dimensionless speed $C$, cf. Figs. 11 and 12, are obtained for pair of ratios $\left(E_{2} / E_{1}=1, \quad \rho_{2} / \rho_{1}=0.01\right)$, i.e. for a periodic shell with a very strong inertial heterogeneity and with elastic homogeneous structure. The smallest values of this speed are obtained for pair of ratios $\left(E_{2} / E_{1}=0.01, \rho_{2} / \rho_{1}=1\right)$, i.e. for a periodic shell with a very strong elastic heterogeneity and with inertial homogeneous structure.

5. For a homogeneous isotropic shell, expression (38) leads to result: $c^{2}=E\left[\left(1-v^{2}\right) \rho\right]^{-1}$. For dimensionless wave propagation speed $C_{\text {hom }}^{2}$ defined by: $C_{\text {hom }}^{2} \equiv \rho(E)^{-1} c^{2}$, we obtain $C_{\text {hom }}^{2}=\left(1-v^{2}\right)^{-1}$. For $v=0.3$, the value of speed $C_{\text {hom }}$ is equal 1.05. Comparing this result with results shown in Figs. 11 and 12, we conclude that in the unbounded homogeneous isotropic shell, the displacement wave propagates along axial direction with speed which is much smaller, i.e. about 70 times smaller, than the smallest velocity obtained for the periodic shell with a very strong elastic heterogeneity $\left(E_{2} / E_{1}=0.01\right)$ and at the same time with inertial homogeneous structure $\left(\rho_{2} / \rho_{1}=1\right)$.

\section{Final remarks and conclusions}

The following remarks and conclusions can be formulated:

- Thin linearly elastic Kirchhoff-Love-type circular cylindrical shells having a periodic microstructure in circumferential direction (uniperiodic shells) are objects of consideration, cf. Figs. 1 and 2. At the same time, the shells have constant geometrical and material properties in axial direction.

- The new averaged combined asymptotic-tolerance model for the analysis of selected dynamic problems for the uniperiodic cylindrical shells under consideration was derived in Tomczyk [8]. Here, the governing 
equations of this model are recalled and applied for investigations of certain micro-dynamic problems for the shells under consideration. The aforementioned model equations consist of macroscopic (asymptotic) model equations (11) for macrodisplacements $u_{\alpha}^{0}(x, \xi, t), w^{0}(x, \xi, t),(x, \xi) \in \Omega \times \Xi, t \in \mathrm{I}$, derived by means of the consistent asymptotic procedure, cf. Woźniak et al. [7], and of microscopic tolerance (nonasymptotic) model equations (20), (21) for fluctuation amplitudes $Q_{\alpha}(x, \xi, t), V(x, \xi, t)$ formulated by applying the tolerance modelling technique, cf. Woźniak et al. [7]. The tolerance modelling is based on the concept of tolerance relations between points and real numbers related to the accuracy of the performed measurements and calculations. The tolerance relations are determined by the tolerance parameters. Macroand microscopic models are combined together under assumption that in the framework of the asymptotic model the solutions (14) to the problem under consideration are known. Contrary to the starting wellknown governing Eq. (7) of Kirchhoff-Love theory with highly oscillating, non-continuous and periodic coefficients, equations of the asymptotic-tolerance model have constant coefficients depending also on a microstructure size. Hence, this model allows us to describe the effect of a length scale on the dynamic shell behaviour. The resulting combined model equations are uniquely determined by the highly oscillating periodic fluctuation shape functions describing oscillations inside the cell. These functions have to be known in every problem under consideration. Under special conditions imposed on the fluctuation shape functions, we can derive microscopic equations (22)-(24), which are independent of solutions (14) obtained within the macroscopic model. It means that an important advantage of this model is that it makes it possible to separate the macroscopic description of some special problems from their microscopic description. Moreover, Eqs. (22)-(24) involve terms with time and spatial derivatives of fluctuation amplitudes. Hence, these equations describe certain time-boundary-layer and space-boundary-layer phenomena strictly related to the specific form of initial and boundary conditions imposed on the unknown fluctuation amplitudes.

- The main aim of this contribution was to apply micro-dynamic equations (22)-(24), proposed in [8] and recalled here, to study two special micro-dynamic problems for a certain cylindrical shell made of two homogeneous elastic isotropic component materials densely and periodically distributed in circumferential direction, cf. Fig. 2. The first of these problems deals with cell-dependent micro-vibrations. The second one deals with propagation of the long waves related to micro-fluctuations of axial displacements.

- The free micro-vibration frequencies have been determined, cf. Eqs. (31)-(33) and investigated. These frequencies depend on a periodicity cell size. Hence, they cannot be obtained in the framework of the asymptotic models commonly used for investigations of dynamics of periodic shells. The influence of the shell elastic, inertial and geometrical properties on the free micro-vibration frequencies has been analysed. From the numerical example, it follows that the free micro-vibration frequencies decrease with the decreasing of differences between inertial properties of the component materials, i.e. with the increasing of ratio $\rho_{2} / \rho_{1} \in[0.01,1]$, cf. Figs. 5b, 7b, 9b, but they increase with the decreasing of differences between elastic properties of the shell material components, i.e. with the increasing of ratio $E_{2} / E_{1} \in[0.01,1]$, cf. Figs. 5a, 7a, 9a. They also decrease with the decreasing of differences between the period length $\lambda$ and the length dimension $L_{1}$ of the shell midsurface in periodicity direction, i.e. with the increasing of ratio $\lambda / L_{1}$, cf. Figs. 4, 6, 8.

- Some new important results have been obtained analysing the long wave propagation problem related to micro-fluctuations in axial direction. We deal with long waves if condition $\lambda / L<<1$ holds, where $\lambda$ is the characteristic length dimension of the cell and $L$ is the wavelength. It was shown that the tolerance-periodic heterogeneity of the shells leads to exponential waves and to dispersion effects, which cannot be analysed in the framework of the asymptotic models for periodic shells. Moreover, the new wave propagation speed (38) depending on the microstructure size has been obtained and investigated. The influence of the shell elastic, inertial and geometrical properties on this cell-dependent speed has been analysed. From the numerical example, it follows that the values of the wave propagation velocity increase with the decreasing of differences between elastic properties of the shell component materials, cf. Fig. 11, but they decrease with the decreasing of differences between inertial properties of the component materials, cf. Fig. 12. Values of the wave propagation speed decrease with the decreasing of differences between period length $\lambda$ and the wavelength $L \equiv L_{2}$, cf. Fig. 10. The strongest decrease in the speed takes place for $\varepsilon \equiv \lambda / L \in[0.0001,0.001]$. 
Open Access This article is distributed under the terms of the Creative Commons Attribution 4.0 International License (http:// creativecommons.org/licenses/by/4.0/), which permits unrestricted use, distribution, and reproduction in any medium, provided you give appropriate credit to the original author(s) and the source, provide a link to the Creative Commons license, and indicate if changes were made.

\section{References}

1. Lewiński, T., Telega, J.J.: Plates, Laminates and Shells. Asymptotic Analysis and Homogenization. World Scientific Publishing Company, Singapore (2000)

2. Awrejcewicz, J., Krysko, V.A., Zhigalov, M.V., Krysko, A.V.: Mathematical model of a three-layer micro- and nano-beams on the hypotheses of the Grigolyuk-Chulkov and the modified couple stress theory. Int. J. Solids Struct. 117, 39-50 (2017)

3. Settimi, V., Trovalusci, P., Rega, G.: Dynamical properties of a composite microcracked bar based on a generalized continuum formulation. Continuum Mech. Thermodyn. (2019). https://doi.org/10.1007/s00161-019-00761-7

4. Hassani, R., Ansari, R., Rouhi, H.: An efficient numerical approach to the micromorphic hyperelasticity. Continuum Mech. Thermodyn. (2019). https://doi.org/10.1007/s00161-019-00808-9

5. Woźniak, C., Wierzbicki, E.: Techniques in Thermomechanics of Composite Solids. Tolerance Averaging Versus Homogenization. Częstochowa University Press, Częstochowa (2000)

6. Woźniak, C., Michalak, B., Jędrysiak, J., (eds.).: Thermomechanics of heterogeneous solids and structures. Tolerance averaging approach. Lodz University of Technology Press, Lodz (2008)

7. Woźniak, C., et al., (eds.).: Mathematical modelling and analysis in continuum mechanics of microstructured media. Silesian University of Technology Press, Gliwice (Poland) (2010)

8. Tomczyk, B.: Length-scale effect in dynamics and stability of thin periodic cylindrical shells. Scientific Bulletin of the Lodz University of Technology, No. 1166, series: Scientific Dissertations, Lodz University of Technology Press, Lodz (2013)

9. Marczak, J., Jędrysiak, J.: Tolerance modelling of vibrations of periodic three-layered plates with inert core. Compos. Struct. 134, 854-861 (2015)

10. Tomczyk, B., Litawska, A.: Tolerance modelling of dynamic problems for thin biperiodic shells. In: Pietraszkiewicz, W., Witkowski, W. (eds.) Shell structures: theory and applications. CRC Press/Balkema, Taylor \& Francis Group, London (2018) pp. 341-344

11. Tomczyk, B., Litawska, A.: Length-scale effect in dynamic problems for thin biperiodically stiffened cylindrical shells. Compos. Struct. 205, 1-10 (2018)

12. Tomczyk, B., Woźniak, C.: Tolerance models in elastodynamics of certain reinforced thin-walled structures. In: Kołakowski, Z., Kowal-Michalska, K. (eds.) Statics, Dynamics and Stability of Structural Elements and Systems, vol. 2, pp. $123-153$. Lodz University of Technology Press, Lodz (2012)

13. Ostrowski, P., Michalak, B.: A contribution to the modelling of heat conduction for cylindrical composite conductors with non-uniform distribution of constituents. Int. J. Heat Mass Transf. 92, 435-448 (2016)

14. Pazera, E., Jędrysiak, J.: Thermoelastic phenomena in the transversally graded laminates. Compos. Struct. 134, 663-671 (2015)

15. Wirowski, A.: Dynamic behaviour of thin annular plates made from functionally graded material. In: Pietraszkiewicz, W., Kreja, I. (eds.) Shell structures: theory and applications. CRC Press/Balkema, Taylor \& Francis Group, London (2010) pp. 207-210

16. Tomczyk, B., Szczerba, P.: Tolerance and asymptotic modelling of dynamic problems for thin microstructured transversally graded shells. Compos. Struct. 162, 365-373 (2017)

17. Tomczyk, B., Szczerba, P.: Combined asymptotic-tolerance modelling of dynamic problems for functionally graded shells. Compos. Struct. 183, 176-184 (2018)

18. Tomczyk, B., Szczerba, P.: A new asymptotic-tolerance model of dynamic and stability problems for longitudinally graded cylindrical shells. Compos. Struct. 202, 473-481 (2018)

19. Kaliski, S.: Vibrations. PWN-Elsevier, Warsaw-Amsterdam (1992)

20. Bensoussan, A., Lions, J.L., Papanicolau, G.: Asymptotic Analysis for Periodic Structures. North-Holland Publishing Co., Amsterdam (1978)

21. Jikov, V.V., Kozlov, C.M., Olejnik, O.A.: Homogenization of Differential Operators and Integral Functionals. Springer, Berlin (1994)

22. Jędrysiak, J.: A contribution to the modelling of dynamic problems for periodic plates. Eng. Trans. 49, 65-87 (2001)

Publisher's Note Springer Nature remains neutral with regard to jurisdictional claims in published maps and institutional affiliations. 